\title{
Exercise Rehabilitation and/or Astragaloside Attenuate Amyloid-Beta Pathology by Reversing BDNF/TrkB Signaling Deficits and Mitochondrial Dysfunction
}

\section{Yu-Ling Wang}

Chi Mei Medical Center

Chung-Ching Chio

Chi Mei Medical Center

Shu-Chun Kuo

Chi Mei Medical Center

Chao-Hung Yeh

Chi Mei Medical Center

Jui-Ti Ma

Chi Mei Medical Center

Wen-Pin Liu

Chi Mei Medical Center

Mao-Tsun Lin

Chi Mei Medical Center

Kao-Chang Lin

Chi Mei Medical Center

Ching-Ping Chang ( $\square$ jessica.cpchang@gmail.com )

Chi Mei Medical Center https://orcid.org/0000-0003-0890-9414

Research article

Keywords: Alzheimer's disease, exercise rehabilitation, astragaloside, BDNF, mitochondrial dysfunction, amyloid-beta

Posted Date: August 2nd, 2021

DOl: https://doi.org/10.21203/rs.3.rs-746587/v1

License: @ (i) This work is licensed under a Creative Commons Attribution 4.0 International License. Read Full License 
Version of Record: A version of this preprint was published at Molecular Neurobiology on March 9th, 2022. See the published version at https://doi.org/10.1007/s12035-022-02728-3. 


\section{Abstract}

Background: Although there are numerous investigations regarding the beneficial effects of exercise rehabilitation (ER) or astragaloside (AST) after amyloid-beta (Ab) pathology, the mechanisms are still not well understood. We aim to assess whether ER and/or AST counteract Ab pathology via diminishing brain-derived neurotrophic factor (BDNF) signaling deficits and mitochondrial dysfunction.

Methods: $A b_{1-42}$ were microinjected into the bilateral ventricles to induce $A b$ neuropathology in rats. The Alzet osmotic pump containing full of AST was implanted subcutaneously during surgery. The ER group of rats started at seven days post-surgery and lasted for four weeks. The ANA12 was administrated once per day to the endpoint of the experiments to antagonize the BDNF action. Neurobehavioral functions were evaluated by $Y$-maze, radial maze, and rotarod tests one day before surgery and 14 to 35 days postsurgery. Cortical and hippocampal expressions of both BDNF/TrkB and cathepsin D were determined by Western blotting method. The rat primary cultured cortical neurons were incubated with BDNF and/or AST and ANA12 followed by exposure to aggregated Ab for 24 hours. The cell viability (by MTT assay), mitochondrial membrane permeability and electrochemical potential (by JC-1 stain), DNA fragmentation (sub-G1 and DNA ladder assay), synaptic plasticity (by immunofluorescence stain), and pTrkB/pAkt/pGSK3b/b-catenin signaling (by Western blot) were determined.

Results: ER and/or AST reversed neurobehavioral disorders, downregulation of cortical and hippocampal expression of both BDNF/TrkB and cathepsin D, neural pathology, Ab accumulation, and altered microglial polarization caused by Ab. In vitro studies also confirmed that topical application of BDNF and/or AST reversed the Ab-induced cytotoxicity, apoptosis, mitochondrial distress, synaptotoxicity, and decreased expression of p-TrkB, AKT, p-GSK3b, and b-catenin in altered rat cortical neurons. In particular, the beneficial effects of combined ER (or BDNF) and AST therapy in vivo and in vitro were superior to ER (or BDNF) or AST alone. Furthermore, we observed that any gains from ER (or BDNF) and/or AST could be significantly eliminated by ANA-12, a potent BDNF/TrkB antagonist.

Conclusion: These results indicate that whereas ER (or BDNF) and/or AST attenuate Ab pathology by reversing BDNF/TrkB signaling deficits and mitochondrial dysfunction. In particular, AST can be an alternative therapy to replace ER.

\section{Background}

Alzheimer's disease (AD) is the most common form of age-related memory loss. It is characterized by cognitive deficits, $\beta$-amyloid $(A \beta)$ deposition, neurodegeneration, neurofibrillary tangle formation, and central neuroinflammation [1]. The exercise provided cognitive benefit in a mouse model of $A D$ by elevating hippocampal and cortical levels of brain-derived neurotrophic factor (BDNF). The hippocampus, parietal cortex, temporal cortex, and frontal cortex of AD brains had substantially reduced BDNF levels [2]. Exercise rehabilitation (ER) shares with BDNF therapy the similar beneficial effects on cognition in an AD's mouse model [1]. 
The astragaloside (AST), a small-molecule saponin purified from Astragalus membranaceus, also shares with BDNF administration the similar beneficial effects in preventing the occurrence of memory loss, synaptotoxicity, oxidative stress, and mitochondrial dysfunction in rat brains caused by A $\beta$ deposition [37]. Again, the neurodegeneration mechanisms underlying the beneficial effects of ER and/or AST on AD conditions remaining unclear.

It has been promoted that abnormal precursor protein (APP) processing and A $\beta$ production in mitochondria disturbed the axonal transport by impairing mitochondrial function and attenuate BDNFneurotrophic tyrosine receptor kinase 2 (TrkB) signaling subsequently [8]. Evidence has accumulated to indicate that $A \beta$ overproduction as a causative event in $A D$ pathogenesis. The present study aimed to investigate whether ER and/or AST attenuate AD pathology via reversing $A \beta$-induced mitochondrial dysfunction and BDNF/TrkB signaling deficits.

To dear with the question, we used bilateral intracerebroventricular $A \beta$ microinjected [3] to ascertain whether the $A \beta$-induced neurobehavior deficits, neuropathology, and altered BDNF/TrkB signaling can be affected by ER and/or AST. Additionally, we used cultured primary cortical neurons in vitro model to confirm whether local application of BDNF ( to mimic ER effects) and/or AST can inhibit A $\beta$-induced cytotoxicity, apoptosis, synaptotoxicity, mitochondrial dysfunction and altered BDNF/TrkB signaling. Furthermore, we would elucidate whether the proposed gains from ER (or BDNF application) and/or AST in reducing $A \beta$-induced $A D$ condition can be eliminated by inhibition of BDNF/ tyrosine kinase receptor $B$ (TrkB) signaling with ANA-12 [9]. Figure 1 depicts the schematic diagrams showing the in vivo and in vitro experimental designs.

\section{Materials And Methods}

All authors had access to the study data and reviewed and approved the final manuscript. All in vivo studies represent 20 animals per group. All in vitro studies are from at least three replicate experiments. All animal experiments were approved and carried out in accordance with the Institutional Animal Care and Use Committee at the Chi Mei Medical Center, Tainan, Taiwan (approved no. 108120110).

\section{Animals}

The laboratory animals were male Wistar rats with a weight of 250-350 g (BioLASCO Taiwan Co., Ltd.). The rats were housed in an air-conditioned animal facility at $26 \pm 0.5^{\circ} \mathrm{C}$ under a 12-hour light-dark cycle and given unlimited access to water and food. All experiments were conducted during the daytime 10:0018:00.

The rats were randomly assigned into eight groups, including (1) sham operation with the sedentary and vehicle-treated group (Sham+Sed+Veh); (2) bilateral intracerebroventricular (i.c.v.) injection of $A \beta_{1-42}$ with the sedentary and vehicle-treated group ( $A b+S e d+V e h)$; (3) i.c.v. injection of $A b_{1-42}$ with $E R$, and vehicletreated group (Ab+ER+Veh); (4) i.c.v. injection of $A b_{1-42}$ with ER and ANA12 administration (Ab+ER+ANA12); (5) i.c.v. injection of $A b_{1-42}$ with astragaloside (AST) and vehicle administration (Ab+AST+Veh); (6) 
i.c.v. injection of $A b_{1-42}$ with AST and ANA12 administration (Ab+AST+ANA12); (7) i.c.v. injection of $A b_{1-}$ 42 with ER and AST combined therapy and vehicle administration (Ab+ER+AST); and (8) i.c.v. injection of $A b_{1-42}$ with ER and AST and ANA12 administration (Ab+ER+AST+ANA12). The ALZET osmotic pump full of AST was implanted subcutaneously. The ANA12 was injected intraperitoneally every day from day 8 to day 35. The treadmill ER was performed on day eight post-surgery. The cognitive functions were measured before surgery and every seven days of post-surgery. After the last cognitive evaluation at week- 5 of post-surgery, the rats were sacrificed.

\section{$A \beta_{1-42}$ oligomerization}

$A \beta_{1-42}$ was obtained from Anaspec (Cat.\# AS-20276, Fremont, CA, USA). The lyophilized A $\beta$ peptides were prepared by reconstituting the powder in $1 \%$ ammonium hydroxide. Subsequently, this stock was dissolved in 1X PBS to obtain a $30 \mathrm{uM}$ working solution and then subjected to oligomerization. For oligomerization, $A \beta$ peptides were incubated at $4{ }^{\circ} \mathrm{C}$ overnight and then aliquoted to store at $-80^{\circ} \mathrm{C}$ until use.

\section{ICV injection of $A \beta_{1-42}$}

Rats were anesthetized with a combination of Zoletil ( $40 \mathrm{mg} / \mathrm{kg}$; Virbac, Nice, France), xylazine hydrochloride (2 mg/kg; Balanzine, Health-Tech Pharmaceutical Co., Taipei, Taiwan), and atropine sulfate (1 mg/kg; Tai Yu Chemical \& Pharmaceutical Co. Ltd., Hsinchu, Taiwan) intraperitoneally (i.p.) and then placed in a standard stereotaxic apparatus (David Kopf Instruments, Tujunga, CA, USA). A middle sagittal incision was made in the scalp and was sterilized using standard procedures. Bilateral holes were drilled in the skull using a dental drill over the lateral ventricles. Rats were bilaterally and intracerebroventricularly (i.c.v., AP: $-0.8 \mathrm{~mm}, \mathrm{ML}: \pm 1.4 \mathrm{~mm}$, DV: $-4.0 \mathrm{~mm}$ ) injected with $20 \mu \mathrm{g} / \mu \mathrm{l} \mathrm{A} \beta_{1-42}$ (Cat.\# AS-20276, AnaSpec, Inc., Fremont, CA, USA) at a rate of $60 \mu \mathrm{l} / \mathrm{hr}$ by using Hamilton microsyringe and a minipump (Hamilton, Reno, NV, USA). The syringe was removed 5 min after injection. The sham group received sterile 1x PBS. After surgery, the scalp was sutured, and sulfamethoxazole was sprinkled on the wound to prevent infection. In addition, penicillin $(40,000 \mathrm{U})$ was injected intramuscularly into the gluteus once a day for 3 days $[10,11]$.

\section{Treadmill exercise protocol}

On day-8 of post-surgery, we trained the rats on a treadmill (model Exer-3/6, Columbus Instruments, Columbus, $\mathrm{OH}, \mathrm{USA}) 5$ days a week for four weeks. They were acclimatized to run for $15 \mathrm{~min}$ at $20 \mathrm{~m} / \mathrm{min}$ at $0^{\circ}$ for 3 days initially, and then they were running for $30 \mathrm{~min}$ at $20 \mathrm{~m} / \mathrm{min}$ at $10^{\circ}, 30 \mathrm{~min}$ at $30 \mathrm{~m} / \mathrm{min}$ at $10^{\circ}, 60 \mathrm{~min}$ at $30 \mathrm{~m} / \mathrm{min}$ at $10^{\circ}$, and $60 \mathrm{~min}$ at $30 \mathrm{~m} / \mathrm{min}$ at $10^{\circ}$ after $1,2,3$, and 4 weeks of training, respectively. Rats in the Sham + Veh and $A \beta+$ Veh groups were placed daily on a stationary treadmill [12].

\section{Drug Treatment}


Astragaloside (AST, molecular formula: $\mathrm{C} 28 \mathrm{H} 32017$; molecular weight $=640.55$, was purchased from Fusol Material Co., Ltd, Tainan, Taiwan) was dissolved in $2 \mathrm{ml}$ of saline with $5 \% \mathrm{EtOH}$ and administered through an ALZET osmotic minipump (Alzet 2ML4; Alza, Palo Alto, CA, USA) at a dose of $40 \mathrm{mg} / \mathrm{kg} /$ day after $\mathrm{Ab}_{1-42}$ injection $[3,13,14]$.

The rats received another i.p. injection of vehicle (1\% dimethylsulfoxide in physiological saline, $1 \mathrm{ml} / \mathrm{kg}$; Sigma-Aldrich, St. Louis, MO, USA) or N-[2-[((Hexahydro-2-oxo-1H-azepin-3-yl) amino]carbonyl] phenyl] benzo [b]thiophene-2-carboxamide (ANA12, a TrkB receptor antagonist, $1 \mathrm{mg} / \mathrm{kg} / \mathrm{day}$, i.p.; Tocris Bioscience, Bristol, UK), respectively [9].

\section{Behavioral tests}

\section{Radial arm maze task}

The maze comprised of 8 arms, extending radially from a central area. Before the training, rats were placed to explore the maze for $5 \mathrm{~min}$ and consume food freely. They were trained for five days to run to the end of the arms and consumed the baited food. The training trial continued until 5 min has passed. After adaption, each animal was checked for working and reference memory, in which the same four arms (no. 2, 4, 6, and 8) were baited. All the rats were trained with one trial: (1) before $A \beta_{1-42}$ injection per day for five days; and (2) after $A \beta_{1-42}$ injection 7, 14, 21, 28, and 35 days. Finally, the number of long-term memory (reference memory) errors (entrance into an arm that never baited) and short-term memory (working memory) errors (repeat entrance into an arm) were counted [15]. Latency was measured as the time elapsed from the beginning to the end of each trial. All measures were averaged across each trial.

\section{Y maze task}

Short-term spatial recognition memory was assessed by analysis of spontaneous alternation in Y-maze, as reported before $[15,16]$. In brief, each rat was placed at the end of one arm and allowed to move through the arms for $8 \mathrm{~min}$, and a sequence of arm entries was recorded. Alternation was obtained as successive entries into the three arms on overlapping triplet sets. The maximum number of possible spontaneous alternations was obtained as the total number of arms entered minus 2 , and the alternation was calculated as the ratio of actual to possible alternations $\times 100$.

\section{Rotarod motor coordination test}

Rotarod accelerating test was also performed on each animal to evaluate motor coordination impairment [17]. It was to examine the possible defects in neuromuscular coordination that might occur on the $A \beta_{1-42}$ injection rats. Before the stereotaxic surgery, each rat was placed in a rotarod apparatus and subjected to an accelerating test. The rat was placed on the rotating rod (at the slowest speed, $4 \mathrm{rpm}$ ) for 1 minute and acceleration to its maximum speed of $30 \mathrm{rpm}$ at $3 \mathrm{~min}$. Each rat that could not hold at the acceleration rod for more than 1 minute was excluded from the further experiments. For the qualified rats starting from the 2 nd day before surgery, the rats were trained per day as described above for 2 days. On 
days $7,14,21,28$, and 35 , after surgery, the rotational speed of the rod was then accelerated to its maximum speed of $30 \mathrm{rpm}$. The length of the time that rats could grasp at the rod was measured. The test score is the average number of seconds that rats could hold onto the rod per trial.

\section{Histological analyses}

Formalin-fixed brains were embedded in paraffin blocks. Serial sections through the hippocampus and cortex were stained with hematoxylin and eosin for microscopic examination. An examiner who was blinded to the experimental conditions evaluated the extent of neuronal damage for each section. The damage scores were determined by two grading systems. In the first system, Honório et al. [18] determined that damage scores from 0 to 4 in which " 0 ", "1", "2", " 3 ", and " 4 " denote no pathological changes, lesions involving $25 \%$ of the field, lesions involving $25 \%$ to $50 \%$, lesions involving $50 \%$ to $75 \%$, and lesions involving $75 \%$ to $100 \%$, respectively. In another system, Liu et al. [19] used 0, 1, 2, and 3 denote normal morphology, minor damage (edema, few pyknotic cells), moderate damage (structural disorganization, edema, moderate pyknotic cells, vacuolization, inflammatory cell infiltration), intense damage (structural disorganization, edema, intense pyknotic cells, vacuolization, inflammatory cell infiltration), respectively. In our present study, we multiplied these two grading scores to present damage scores.

\section{$A \beta$ Plaque stain}

Amyloid plaques were stained with Thioflavin-S. The deparaffinized and hydrated sections were incubated in $0.25 \%$ potassium permanganate solution for $20 \mathrm{~min}$, rinsed in distilled water, and incubated in bleaching solution ( $2 \%$ oxalic acid and $1 \%$ potassium metabisulfite) for $2 \mathrm{~min}$. After rinsed in distilled water, the sections were transferred to a blocking solution ( $1 \%$ sodium hydroxide and $0.9 \%$ hydrogen peroxide) for $20 \mathrm{~min}$, incubated for $5 \mathrm{~s}$ in $0.25 \%$ acidic acid, washed in distilled water, and stained for 5 min with $0.0125 \%$ Thioflavin-S in $50 \%$ ethanol. The sections were washed with $50 \%$ ethanol, placed in distilled water, and then covered with a glass cover using a mounting solution [20].

\section{Triple immunofluorescence staining}

Dissected brains were fixed in $4 \%$ phosphate-buffered formalin at $4{ }^{\circ} \mathrm{C}$, and subjected to immunohistochemical staining. The following antibodies were used: Iba1 (1:200; \#GTX10042, GeneTex Inc., Irvine, CA, USA), CD86 (1:200, \#ab53004, Abcam Inc., Boston, MA, USA), and Neu-N (1:200, MAB377, Merck Millipore, Billerica, MA, USA). The staining was visualized using Alexa Fluor 568- and Alexa Fluor 488-conjugated secondary antibodies (1:500, Invitrogen, Carlsbad, CA, USA) and 4', 6-diamidino-2 phenylindole (DAPI, 1:5000, \#D1306, Invitrogen).

For degenerative neuron detection, after secondary antibody incubation, the brain tissue slides were immersed in a solution of $5 \%$ sodium hydroxide and $100 \%$ ethanol for $5 \mathrm{~min}$, followed by immersion in $70 \%$ ethanol for $2 \mathrm{~min}$, distilled water for $2 \mathrm{~min}$, and $0.06 \%$ potassium permanganate solution for $10 \mathrm{~min}$. The sections were then rinsed in distilled water for $2 \mathrm{~min} / 2$ times and placed in a $0.0004 \%$ Fluoro-Jade B 
solution made by adding $4 \mathrm{ml}$ of a $0.01 \%$ stock solution of Fluoro-Jade B to $96 \mathrm{ml}$ of $0.1 \%$ acetic acid. After 20 min in the Fluoro-Jade B staining solution, the stained slides were thoroughly washed in distilled water, dehydrated, and coverslipped [21, 22].

Before the primary antibody incubation, the brain slides were treated with proteinase $\mathrm{K}(20 \mu \mathrm{g} / \mathrm{ml})$ for 15 min at room temperature for neuronal apoptosis detection. Subsequently, equilibration buffer was applied for $10 \mathrm{sec}$, and the brain slides were immersed and incubated for $1 \mathrm{~h}$ in working strength terminal deoxynucleotidyl transferase $(\mathrm{TdT})$ enzyme solution at $37^{\circ} \mathrm{C}$. Following incubation in stop/wash buffer for 10 min to terminate the reaction, brain slides were incubated for $30 \mathrm{~min}$ in working strength antidigoxigenin conjugate at room temperature in the dark to visualize the DNA fragments. Proteinase K, equilibration buffer, and stop/wash buffer were all included in the terminal deoxyribonucleotide transferase-mediated dUTP nick end labeling (TUNEL) assay kit (\#630108, Takara Bio USA, Inc., CA, USA) [23]. TUNEL-positive neurons with condensed nuclei were identified as dead neurons.

After a final wash with PBS, slides were mounted in glycerol gelatine mounting medium (\#GG1-15 ML, Sigma-Aldrich, St. Louis, MO, USA) and viewed using an upright fluorescence microscope (Carl Zeiss Microscopy $\mathrm{GmbH}$, Jena, Germany) at excitation/emission wavelengths of 578/603 nm (rhodamine, red) and 490/525 nm (FITC, green). A digital camera linked to a computer running Axioscope version 4 (Carl Zeiss) was used to capture images. A pathologist counted the percentage of Fluoro-Jade+NeuN/DAPI and TUNEL+NeuN/DAPI double-labeled cells in 6 fields per section in cortex and hippocampus (x 400 magnification).

\section{Western blot assay}

Western blotting was performed by using the standard method. The total proteins, obtained from cells cultures or brain tissues, were extracted by the modified RIPA buffer (50mM Tris-HCl, pH7.4, 1\% NP-40, $0.25 \%$ Na-deoxycholate, $150 \mathrm{mM} \mathrm{NaCl}, 1 \mathrm{mM}$ EDTA) containing protease and phosphatase inhibitors (Sigma, St Louis, Mo, USA). Protein concentrations were quantified by the Bradford method (Bio-Rad, Hercules, CA, USA). For blot analysis, protein extracts were boiled for 10 min in loading buffer, separated on SDS-PAGE, and transferred to polyvinylidene difluoride membrane (Pall Corporation, East Hills, NY, USA) using a wet transfer system (Bio-Rad, Hercules, CA, USA). The membranes were blocked in $5 \%$ nonfat milk in PBS containing $0.05 \%$ Tween-20 (TBS-T) for $1 \mathrm{hr}$ at room temperature. The membranes were hybridized with Cathepsin D (\#SC-46656, Santa Cruz Biotechnology, Santa Cruz, CA, USA), BDNF (\#ab108319, Cell Signaling Technology, Inc., Beverly, MA, USA), TrkB (\#4603, Cell Signaling), PhosphoTrkB (\#ABN1381, Millipore), Phospho-Akt (phosphoresidue serine 473, \#4060, Cell Signaling), total Akt (\#9272, Cell Signaling), Phospho-GSK-3 $\beta$ (phosphoresidue serine 9, \#9336, Cell Signaling), total GSK-3 $\beta$ (\#9315, Cell Signaling), b-catenin (\#9562, Cell Signaling), and $\beta$-actin (\#SC-47778, Santa Cruz) antibodies for overnight at $4^{\circ} \mathrm{C}$. After washing with TBS-T, the membranes were continuously incubated with appropriate secondary antibodies coupled to horseradish peroxidase (Cell Signaling Technology, Inc.) for $1 \mathrm{hr}$ at room temperature. The blots were developed in the ECL Western detection reagents (PerkinElmer, 
Waltham, MA, USA) and exposed to Hyperfilm ECL (GE Healthcare, Piscataway, NJ, USA). Protein bands were scanned and quantified using ImageMaster TotalLab image analysis software (GE healthcare).

\section{Ab-induced primary cortical neuron injury model}

Neuronal cultures were prepared from the cortex of embryonic day 18 rat embryos. In brief, embryos were removed and their brain cortices dissected. The neurons were obtained by digestion of papain $(2 \mathrm{mg} / \mathrm{ml}$ for 30 min at $30{ }^{\circ} \mathrm{C}$, Millipore, Billerica, MA, USA) followed by trituration with a $1 \mathrm{ml}$ pipette. The cells were plated onto poly-L-lysine (Trevigen, Gaithersburg, MD, USA)-coated 6 well plates or dishes and maintained in neurobasal media supplemented with 2\% B27 and $0.5 \mathrm{mM}$ GlutaMax (Invitrogen, Carlsbad, CA, USA) at $37^{\circ} \mathrm{C}$ in a humidified $5 \% \mathrm{CO}_{2}$ incubator. Culture media were exchanged every 4 days, and cells were grown for 8 days in vitro (DIV) before $A \beta_{25-35}$ treatment. $A b_{25-35}$ was purchased from Kelowna International Scientific Inc. (Taipei, Taiwan) and dissolved in water with vortex. The solution was allowed to aggregate for 6 days at $37^{\circ} \mathrm{C}$ before use. BDNF protein obtained from GenScript (Piscataway, NJ, USA) was dissolved $(100 \mathrm{ug} / \mathrm{ml})$ in distilled water. The Chinese herb Astragaloside (AST) was made up as a $100 \mathrm{mg} / \mathrm{ml}$ stock in DMSO. ANA12 (TrkB selective antagonist) was purchased from Selleckchem (Houston, TX, USA) and dissolved in DMSO. To induce cell cytotoxicity, the cortical neurons were exposed

to $60 \mathrm{uM} \mathrm{Ab}{ }_{25-35}$ for $24 \mathrm{~h}$. BDNF, AST, and ANA12 were administered at $400 \mathrm{ng} / \mathrm{ml}, 400 \mathrm{ug} / \mathrm{ml}$, and $35 \mathrm{uM}$, respectively.

\section{MTT assay for cell viability}

Cell viability was determined by 3-(4,5-Dimethylthiazol-2-yl)-2,5-diphenyltetrazolium bromide (MTT) assay. After treatment, cells were treated with the MTT solution (final concentration, $5 \mu \mathrm{g} / \mathrm{ml}$ ) for $2 \mathrm{~h}$. The dark blue formazan crystals formed in intact cells were solubilized with lysis buffer ( $20 \%$ sodium dodecylsulfate in $50 \%$ aqueous $\mathrm{N}, \mathrm{N}$-dimethylformamide). The absorbance of the sample was read at 540-595 nm with a MultiSkan GO microplate reader (Thermo Fisher Scientific, MA, USA). Results were expressed as the percentage (\%) of MTT reduction, assuming that the absorbance of control cells was $100 \%$.

\section{Synaptic density}

The cells were fixed with $4 \%$ paraformaldehyde in PBS, permeabilized, and blocked with $0.3 \%$ Triton-X 100 plus and $5 \%$ normal bovine serum in PBS. The cultures were then incubated with combinations of the primary antibodies against the following targets: microtubule-associated protein 2 (MAP-2; a neuronal marker; Santa Cruz) and synaptophysin (is an integral membrane protein localized to synaptic vesicles; Santa Cruz) and diluted in $5 \%$ BSA overnight at $4{ }^{\circ} \mathrm{C}$. Then, cortical neurons were incubated with the appropriate secondary antibodies (Alexa Fluor 568-conjugated goat anti-mouse IgG or Alexa Fluor 488conjugated goat anti-mouse) for $1 \mathrm{~h}$ at room temperature. The neurons were also stained with DAPI (Sigma-Aldrich) as a nuclear marker. Their digital images were captured with a 20x objective (N.A. 0.75) by a fluorescence microscope system (Carl Zeiss Microscopy GmbH, Jena, Germany) with Zen Software (Carl Zeiss). Synaptic density was measured as a synaptophysin-positive area. At least 10 fields obtained 
from different experiments were conducted in triplicate from 3 cultures, were captured and measured for each condition. Values obtained for each field $\left(0.15 \mathrm{~mm}^{2}\right)$ were pooled to obtained mean values for each culture.

\section{Mitochondrial membrane potential analysis}

After culturing, JC-1 (5,5',6,6'-Tetrachloro-1,1',3,3'-tetraethyl-imidacarbocyanine iodide; BD Mitoscreen kit, San Jose, CA, USA) was added to a final concentration of $2.5 \mathrm{mM}$, and cells were shaken in the dark at 37 ${ }^{\circ} \mathrm{C}$ for $15 \mathrm{~min}$. Following incubation, $400 \mathrm{ul}$ staining buffer was added to each sample and analyzed on the Novocyte flow cytometry (ACEA Biosciences, CA, USA). A total of 10,000 events were measured using FL-1 channel $(525 \mathrm{~nm})$ and results were expressed as a percentage.

\section{Sub-G1 phase analysis}

At the end of the treatment period, the cells were harvested, washed with PBS, and fixed in cold $70 \%$ ethanol at $-20^{\circ} \mathrm{C}$ overnight. The fixed cells were washed with PBS and treated with propidium iodide (PI) staining solution containing $10 \mathrm{ug} / \mathrm{ml} \mathrm{PI} \mathrm{(ThermoFisher} \mathrm{Sci)} \otimes 100 \mathrm{ug} / \mathrm{ml}$ RNase (ThermoFisher Sci) and $0.1 \%$ Triton X-100 (Sigma-Alrich) for $30 \mathrm{~min}$ at $37^{\circ} \mathrm{C}$. Finally, the samples were analyzed in the Novocyte flow cytometer using NovoExpress software to analyze sub-G1 distribution.

\section{Evaluation of DNA fragmentation}

DNA was extracted by the use of a commercially available Wizard genomic DNA purification kit (Promega, Madison, WI, USA) following the manufacturer's instructions. Neuron cell was lysed in Nuclei lysis solution and then digested all RNAs with RNase A solution for 30 min at $37^{\circ} \mathrm{C}$ water bath. Protein precipitation solution was added, vortexed vigorously, and kept on ice for $5 \mathrm{~min}$. After short centrifugation, the supernatant was transferred to a new microtube containing $600 \mathrm{ul}$ isopropanol, mixed by inversion, and centrifuged. The supernatant was removed, and the pellet was washed in $600 \mu \mathrm{l} 70 \%$ ethanol. Finally, the pellet was air-dried, then rehydrated with hydration solution, and determined the DNA concentration. Two ug of extracted DNA was loaded to $1.8 \%$ agarose gel electrophoresis in Tris-borateEDTA buffer system, stained with ethidium bromide, and visualized through a Quantum CX5 UVtransilluminator (Vilber Lourmat, Torcy, France) and photographed to determine the DNA concentration.

\section{Statistics}

The person charged with functional outcome measurements was the only one that was blinded to treatments among those working on animals (single-blind). She used cage and animal codes to recognize individuals and to report repeated measurements on data collection forms. Statistical analyses were performed using GraphPad Prism 7.01 (GraphPad Software Inc., CA, USA). Parameters such as histological scores and the immunofluorescence staining data with non-normal distribution were analyzed by the Kruskal-Wallis test with Dunn's post-hoc test. One-way analysis of variance (ANOVA) with Tukey's post hoc test was used by an investigator blinded to the treatment groups and the flow cytometry 
and Western blotting data. We performed two-way ANOVA with Tukey's multiple comparisons tests to analyze behavioral performance data. A Student's t-test was used to compare variables for two groups if there was a significant difference. All data were expressed as mean \pm standard deviation (SD). P-values < 0.05 were considered statistically significant.

\section{Results}

\section{ANA-12 can ameliorate the beneficial effects of ER and/or AST on Ab-induced cognitive and motor deficits in rats}

Thirty-five days following Ab injections, rats displayed deficits in working memory (e.g., decreased \% of alternations in Y-maze test; Figure 2A), spatial reference memory (e.g., increased latency time, increased number of reference memory errors, and increased number of working memory errors in the radial-maze test; Figure 2B, 2C, and 2D), and motor performances (e.g., decreased latency time and decreased RPM counts in Rotarod test; Figure 2E and 2F). In rats, the Ab-induced cognitive and motor deficits were significantly reduced by ER and/or AST therapy (Figure 2). The beneficial effects of ER and/or AST therapy can be significantly attenuated by ANA12 (Figure 2).

\section{ANA12 can attenuate the beneficial effects of ER and/or AST on Ab-induced neuronal degeneration, apoptosis, and $A b$ accumulation and altered microglia polarization in rats}

Rats with intracerebroventricularly microinjected $A b$ displayed neuronal degeneration (as revealed by hematoxylin-eosin staining [Figure 3A and 3B], and NeuN with Fluoro-Jade B staining [Figure 3C and 3D]), apoptosis (as revealed by NeuN with TUNEL staining; Figure 4A and 4B), Ab accumulation (as revealed by thioflavin-S staining; Figure 4C and 4D), and altered microglia polarization in cortical and hippocampal regions of rat brain (as revealed by Iba- 1 and CD86 co-positive cells; Figure 5). All of the above-mentioned parameters caused by Ab injections could be significantly reversed by ER and/or AST therapy (Figure 35). Again, any beneficial effects from ER and/or AST could be significantly counteracted by ANA12 therapy (Figure 3-5).

\section{ANA12 can reverse the beneficial effects of BDNF and/or AST on Ab-induced morphology changes, cytotoxicity, apoptosis, mitochondria distress, and synaptotoxicity in cultured rat cortical neurons}

Local application of BDNF and/or AST also inhibited Ab-induced morphological changes (Figure 6 A), cytotoxicity (e.g., decreased cell viability, Figure 6 B), apoptosis (e.g., increased sub-G1 and DNA fragmentation, Figure $6 \mathrm{C}$-E), increased mitochondrial membrane permeability and consequent loss of electrochemical potential (e.g., increased percentage of JC-1 green fluorescence positive cell numbers, Figure 6 F-G), and synaptotoxicity (e.g., increased loss of both a dendritic marker, microtubule-associated protein-2 (MAP-2) and synaptic-protein, synaptophysins) in cultured primary rat cortical cells (please see Figure 7 A and 7 B). The beneficial effects of BDNF and/or AST were all eliminated by ANA12 therapy. 
ANA12 can reverse the beneficial effects of BDNF (or ER) and/or AST on Ab-induced p-TrkB, p-Akt, pGSK3b, and b-catenin deficits in cultured cortical neurons and reduced cortical and hippocampal levels of $\mathrm{BDNF} / \mathrm{TrkB} /$ cathepsin $\mathrm{D}$ in vivo

Figure $7 \mathrm{C}$ and Figure $7 \mathrm{D}$ showed that BDNF and/or AST therapy inhibited Ab-induced reduced expression of p-TrkB, p-Akt (Ser473), p-GSK3b (Ser9), and b-catenin in cultured primary cortical neurons, which can be wiped out by ANA12. In vivo studies also showed that the Ab-induced decreased cortical and hippocampal expression of BDNF, TrkB, and cathepsin Dcould be reversed by ER and/or AST (Figure $7 \mathrm{E}$ and Figure $7 \mathrm{~F}$ ). Again, the beneficial effects of ER and/or AST therapy on Ab-induced alternations in vivo were all significantly counteracted by ANA-12 application.

\section{Discussion}

Brain $\mathrm{Ab}$ accumulation causes neurological injury via inducing BDNF/TrkB signaling deficits as well as mitochondrial dysfunction

Our in vivo study showed that central injection of Ab oligomers caused motor and cognitive deficits accompanied by decreased expression of both BDNF/TrkB and cathepsin D and neuronal degeneration, apoptosis, Ab accumulation and altered microglial polarization in cortical and hippocampal regions of rat brain. Simultaneously, our in vitro study demonstrated that local application of Ab caused cytotoxicity, apoptosis, mitochondrial distress, synaptotoxicity, and decreased expression of BDNF/TrkB/p-Akt/pGSK3b/b-catenin in cultured cortical neurons. These observations suggest that brain accumulation of Ab oligomers induces BDNF/TrkB signaling deficits and mitochondrial dysfunction which results in neurodegeneration and neurobehavioral disorders in rats.

ER and/or AST therapy attenuate neurological injury via restoration of brain BDNF/TrkB signaling and mitochondrial function in Ab-treated rats

Our in vivo results showed that ER and/or AST might attenuate neurobehavioral disorders and neurodegeneration by restoring the cortical and hippocampal expression levels of BDNF/TrkB and cathepsin D in Ab-microinjected rats. Our in vitro results also showed that topical of BDNF and/or AST might attenuate neurodegeneration via restoring the levels of BDNF/TrkB/p-Akt/p-GSK3b/b-catenin expression in Ab-treated rat cortical neurons. In most of the parameters studied in the present study, the beneficial effects of combined ER (or BDNF) and AST application were superior to ER (or BDNF) or AST alone. The most striking finding is that all the beneficial effects exerted by ER and/or AST in vivo and BDNF an/or AST in vitro were significantly eliminated by ANA12, a potent BDNF/TrkB antagonist therapy. Thus, it can be derived from these observations that whereas ER or AST therapy alone may improve neurodegeneration and neurobehavioral disorders in $A D$ rats via preserving both cerebral BDNF/TrkB/cathepsin D and BDNF/TrkB/p-Akt/p-GSK3b/b-catenin signaling pathways, combining these two treatments may potentiate each other's therapeutic effects.

BDNF/TrkB/cathepsin D signaling deficits in AD pathology

Page $12 / 30$ 
In people with AD [24-27] and in rodent models [28, 29], reduced levels of BDNF and its receptors, TrkB, are related to degeneration of hippocampal and cortical neurons. The increasing of BDNF in the brain with viruses [30,31] or transplanted cells [32-34] results in marked neuroprotective effects in several animal models of AD. In our results, ER and/or AST application might attenuate neurobehavior disorders and neurodegeneration in Ab-microinjected rats by preserving neuronal networks' BDNF-mediated functional integrity [35].

Cathepsin $D$ is essential for lysosomal integrity to degrade misfolded/damaged/ oxidized proteins, including $A \beta$ and tau, to prevent toxic accumulation [36]. Genetic depletion of cathepsin $D$ in mice causes a dramatic accumulation of Ab in lysosomes [37]. They hypothesize that Ab may trigger tauopathy by competitive inhibition of cathepsin D mediated degradation of pathological forms of tau. Our present results showed that ER and/or AST inhibits the accumulation of Ab and tau in the brain and leads to restoration of normal neurobehavioral function.

\section{BDNF/TrkB/p-Akt/p-GSK3b/b-catenin signaling deficits in Ab pathology}

Our in vitro results showed that local application of BDNF and/or AST can attenuate Ab-induced neurodegeneration and reduced expression of BDNF/TrkB/p-Akt/p-GSK3b/b-catenin in cultured rat cortical neurons. Our results are consistent with many investigations describe below. Glycogen synthase kinase 3 beta (GSK3b) has been considered as a putative therapeutic target for promoting functional recovery in the injured or degenerative brain [38]. For example, suppression of GSK3b activity is required for axon formation and elongation, whereas an enhancement of GSK3b activity is related to mitochondrial dysfunction, DNA fragmentation, and cell death [39]. Increased GSK3b activity or activation of GSK3b is linked to the pathogenesis of $A D$ through $A b$ and tau accumulation and mitochondrial distress [40]. Akt has been shown to phosphorylate at serine residue 9 (Ser9) and inactivate GSK3b, leading to stabilized and active b-catenin [38]. The Ser9 phosphorylation results in switching GSK3b from baseline activity to inactive status [38]. Local inactivation of GSK3b would maintain axonal growth and synaptic plasticity [41]. Previous studies established that BDNF activation of TrkB via the phosphoinositide-3-kinase (PI3K)/Akt signaling pathway attenuates the effects of chemotherapy in neuroblastoma cells [42]. Akt (Ser473) and GSK3b (Ser9) phosphorylation was accelerated in the injured cortex and involved neuronal survival after brain injury [43]. Moreover, neuroprotection of b-catenin against brain injury was partly mediated by enhanced and persistent activation of the Akt/GSK3b signaling pathway [43].

\section{Association between mitochondrial dysfunction and $A D$ pathology}

The mitochondrial dysfunction cascade hypothesis holds that amyloid precursor proteins and $A b$ accumulation in the mitochondrial membrane can evoke mitochondrial dysfunction and may initiate the AD pathology subsequently [44]. Our present results showed that topical application of Ab-induced mitochondrial dysfunction as evidenced by increased mitochondrial membrane permeability and consequent loss of electrochemical potential (e.g., increased percentage of JC-1 fluorescence positive cell numbers in the cultured rat cortical neurons). The Ab-induced mitochondrial dysfunction can be 
ameliorated by topical application of BDNF and/or AST. Mitochondrial dysfunction induces BDNF axonal transport deficits [8] and synaptic dysfunction, and neuronal damage[40]. Thus, it can be derived that ER and/or AST may ameliorate neurodegeneration and neurobehavioral disorders in Ab-treated rats via restoration of mitochondrial function.

\section{Association between ANA12 and AD pathology}

Our results showed that ANA12 significantly wiped out the beneficial effects from ER and/or AST therapy in Ab-microinjected rats or in cultured rat cortical neurons. These results indicate that antagonism of cortical and hippocampal BDNF/TrkB signaling with ANA12 significantly alleviates the beneficial effects from ER and/or AST in AD pathology. Our present findings are consistent with a recent investigation showing that ANA12 alleviates the beneficial effects from overexpression of hippocampal BDNF in post-stroke cognitive deficits [45].

\section{Conclusions}

In summary, as depicted in Fig. 8, intracerebroventricularly microinjected $A \beta$ in rats might cause neurodegeneration as well as neurobehavioral disorders as well as neural injury via suppressing cortical and hippocampal levels of BDNF/TrkB/cathepsin D. In vitro studies further confirmed that topical application of $A \beta$ might induce cortical neuronal death via inducing BDNF/TrkB/p-Akt/p-GSK3 $\beta / \beta$-catenin signaling deficits as well as mitochondrial dysfunction. Exercise rehabilitation (or topical application of BDNF) and/or AST therapy might attenuate A $\beta$-induced neurodegeneration as well as neurobehavioral disorders via restoring the cortical and hippocampal levels of BDNF/TrkB signaling and mitochondrial function. Antagonism of BDNF/TrkB with ANA12 wiped out the beneficial effects exerted by ER (or BDNF) and/or AST. Our data indicate that whereas ER and/or AST attenuate $A \beta$ neuropathology by reversing the brain BDNF/TrkB signaling deficits and mitochondrial dysfunction induced by $A \beta$ accumulation, combining these two potentiate each other's therapeutic effects. Furthermore, AST can be an alternative therapy to replace ER.

\section{Abbreviations}
Ab, amyloid-beta

p-Akt, phosphorylated serine/threonine-protein kinase

t-Akt, total form serine/threonine-protein kinase

ANA12, N-[2-[(Hexahydro-2-oxo-1H-azepin-3-yl)amino]carbonyl]phenyl] benzo[b]thiophene-2-carboxamide AST, astragaloside

BDNF, brain-derived neurotrophic factor 
CTSD, cathepsin D

DAPI, 4',6-diamidino-2-phenylindole

ECL, enhanced chemiluminescence

ER, exercise rehabilitation

GSK3b, Glycogen Synthase Kinase-3 beta

i.c.v., intracerebroventricular

i.p., intraperitoneal

Iba-1, ionized calcium-binding adaptor molecule 1

NeuN, neuronal nuclei

PBS, phosphate-buffered saline

RIPA, radioimmunoprecipitation assay buffer

SDS-PAGE, sodium dodecyl sulfate-polyacrylamide gel electrophoresis

p-TrkB, phosphorylated tropomyosin receptor kinase B

t-TrkB, total tropomyosin receptor kinase B

TUNEL, terminal deoxyribonucleotide transferase-mediated dUTP nick end labeling

\section{Declarations}

\section{Ethics approval and consent to participate}

All animal experiments were approved and carried out in accordance with the Institutional Animal Care and Use Committee at the Chi Mei Medical Center, Tainan, Taiwan (approved no. 108120110).

Consent for publication

Not applicable.

\section{Availability of data and materials}

The authors confirm that the data supporting the findings of this study are available within the article and supplementary materials. 
The authors declare that they have no competing interests.

\section{Funding}

This study was supported by the Taiwan Ministry of Science and Technology (MOST) (grant no. MOST109-2314-B-384-002-MY3, MOST106-2314-B-384 -001 -MY3, MOST 105-2314-B-384 -001 -MY3) and Chi Mei Medical Center (Taiwan) grant CMFHT10802. The funders had no role in the study design, data collection, and analysis, the decision to publish, or preparation of the manuscript.

\section{Authors' contributions}

Study concept and design (WYL, SCK, CCC); acquisition of data (CHY, JTM, WPL); analysis and interpretation of data (WYL,SCK, CHY); drafting of the manuscript (CCC, $\mathrm{CPC}, \mathrm{KCL}$ ); critical revision of the manuscript for important intellectual content (SCK, CCC); statistical analysis (WYL, JTM, WPL); material support (CCC, $\mathrm{CPC})$; obtained funding $(\mathrm{CCC}, \mathrm{CPC}, \mathrm{KCL})$. All authors read and approved the final manuscript.

\section{Acknowledgements}

We acknowledge Ms. Meng-Tsung Ho for her excellent editorial assistance in manuscript preparation.

\section{Authors' information (optional)}

No further information given.

\section{References}

1. Choi SH, Bylykbashi E, Chatila ZK, Lee SW, Pulli B, Clemenson GD, et al. Combined adult neurogenesis and BDNF mimic exercise effects on cognition in an Alzheimer's mouse model. Science. 2018; 361. https://doi.org/10.1126/science.aan8821.

2. Allen SJ, Watson JJ, Dawbarn D. The neurotrophins and their role in Alzheimer's disease. Curr Neuropharmacol. 2011; 9:559-73. https://doi.org/10.2174/157015911798376190.

3. Chang CP, Liu YF, Lin HJ, Hsu CC, Cheng BC, Liu WP, et al. Beneficial Effect of Astragaloside on Alzheimer's Disease Condition Using Cultured Primary Cortical Cells Under $\beta$-amyloid Exposure. Mol Neurobiol. 2016; 53:7329-40. https://doi.org/10.1007/s12035-015-9623-2.

4. Polat E, Bedir E, Perrone A, Piacente S, Alankus-Caliskan O. Triterpenoid saponins from Astragalus wiedemannianus Fischer. Phytochemistry. 2010; 71:658-62. https://doi.org/10.1016/j.phytochem.2009.11.013.

5. Li WZ, Wu WY, Huang DK, Yin YY, Kan HW, Wang X, et al. Protective effects of astragalosides on dexamethasone and Aß25-35 induced learning and memory impairments due to decrease amyloid precursor protein expression in 12-month male rats. Food Chem Toxicol. 2012; 50:1883-90. https://doi.org/10.1016/j.fct.2012.03.064. 
6. Tohda C, Tamura T, Matsuyama S, Komatsu K. Promotion of axonal maturation and prevention of memory loss in mice by extracts of Astragalus mongholicus. Br J Pharmacol. 2006; 149:532-41. https://doi.org/10.1038/sj.bjp.0706865.

7. Sun Q, Jia N, Wang W, Jin H, Xu J, Hu H. Protective effects of astragaloside IV against amyloid beta1-42 neurotoxicity by inhibiting the mitochondrial permeability transition pore opening. PLoS One. 2014; 9:e98866. https://doi.org/10.1371/journal.pone.0098866.

8. Ye X, Tai W, Zhang D. The early events of Alzheimer's disease pathology: from mitochondrial dysfunction to BDNF axonal transport deficits. Neurobiol Aging. 2012; 33:1122.e1-10. https://doi.org/10.1016/j.neurobiolaging.2011.11.004.

9. Hsu CC, Kuo TW, Liu WP, Chang CP, Lin HJ. Calycosin Preserves BDNF/TrkB Signaling and Reduces Post-Stroke Neurological Injury after Cerebral Ischemia by Reducing Accumulation of Hypertrophic and TNF-a-Containing Microglia in Rats. J Neuroimmune Pharmacol. 2020. https://doi.org/10.1007/s11481-019-09903-9.

10. Kantar Gok D, Hidisoglu E, Ocak GA, Er H, Acun AD, Yargıcoglu P. Protective role of rosmarinic acid on amyloid beta 42-induced echoic memory decline: Implication of oxidative stress and cholinergic impairment. Neurochem Int. 2018; 118:1-13. https://doi.org/10.1016/j.neuint.2018.04.008.

11. Wong RS, Cechetto DF, Whitehead SN. Assessing the Effects of Acute Amyloid $\beta$ Oligomer Exposure in the Rat. Int J Mol Sci. 2016; 17. https://doi.org/10.3390/ijms17091390.

12. Kim BK, Shin MS, Kim CJ, Baek SB, Ko YC, Kim YP. Treadmill exercise improves short-term memory by enhancing neurogenesis in amyloid beta-induced Alzheimer disease rats. J Exerc Rehabil. 2014; 10:2-8. https://doi.org/10.12965/jer.140086.

13. Chio CC, Lin MT, Chang CP, Lin HJ. A positive correlation exists between neurotrauma and TGF- $\beta 1$ containing microglia in rats. Eur J Clin Invest. 2016; 46:1063-9. https://doi.org/10.1111/eci.12693.

14. Turégano L, Martínez-Rodríguez R, Alvarez MI, Gragera RR, Gómez de Segura A, De Miguel E, et al. Histochemical study of acute and chronic intraperitoneal nicotine effects on several glycolytic and Krebs cycle dehydrogenase activities in the frontoparietal cortex and subcortical nuclei of the rat brain. J Neurosci Res. 2001; 64:626-35. https://doi.org/10.1002/jnr.1116.

15. Baluchnejadmojarad T, Mohamadi-Zarch SM, Roghani M. Safranal, an active ingredient of saffron, attenuates cognitive deficits in amyloid $\beta$-induced rat model of Alzheimer's disease: underlying mechanisms. Metab Brain Dis. 2019; 34:1747-59. https://doi.org/10.1007/s11011-019-00481-6.

16. Ionita R, Postu PA, Beppe GJ, Mihasan M, Petre BA, Hancianu M, et al. Cognitive-enhancing and antioxidant activities of the aqueous extract from Markhamia tomentosa (Benth.) K. Schum. stem bark in a rat model of scopolamine. Behav Brain Funct. 2017; 13:5. https://doi.org/10.1186/s12993017-0123-6.

17. Huang TC, Lu KT, Wo YY, Wu YJ, Yang YL. Resveratrol protects rats from Aß-induced neurotoxicity by the reduction of iNOS expression and lipid peroxidation. PLoS One. 2011; 6:e29102. https://doi.org/10.1371/journal.pone.0029102. 
18. Honório JE, Jr., Vasconcelos GS, Rodrigues FT, Sena Filho JG, Barbosa-Filho JM, Aguiar CC, et al. Monocrotaline: histological damage and oxidant activity in brain areas of mice. Oxid Med Cell Longev. 2012; 2012:697541. https://doi.org/10.1155/2012/697541.

19. Liu X, Gu X, Li Z, Li X, Li H, Chang J, et al. Neuregulin-1/erbB-activation improves cardiac function and survival in models of ischemic, dilated, and viral cardiomyopathy. J Am Coll Cardiol. 2006; 48:1438-47. https://doi.org/10.1016/j.jacc.2006.05.057.

20. Zhang Z, Obianyo O, Dall E, Du Y, Fu H, Liu X, et al. Inhibition of delta-secretase improves cognitive functions in mouse models of Alzheimer's disease. Nat Commun. 2017; 8:14740. https://doi.org/10.1038/ncomms14740.

21. Qin L, Crews FT. NADPH oxidase and reactive oxygen species contribute to alcohol-induced microglial activation and neurodegeneration. J Neuroinflammation. 2012; 9:5. https://doi.org/10.1186/1742-2094-9-5.

22. Shah SA, Yoon GH, Ahmad A, Ullah F, UI Amin F, Kim MO. Nanoscale-alumina induces oxidative stress and accelerates amyloid beta $(A \beta)$ production in ICR female mice. Nanoscale. 2015; 7:15225-37. https://doi.org/10.1039/c5nr03598h.

23. Su C, Chen Y, Chen Y, Zhou Y, Li L, Lu Q, et al. Effect of electroacupuncture at the ST36 and GB39 acupoints on apoptosis by regulating the p53 signaling pathway in adjuvant arthritis rats. Mol Med Rep. 2019; 20:4101-10. https://doi.org/10.3892/mmr.2019.10674.

24. Connor B, Young D, Yan Q, Faull RL, Synek B, Dragunow M. Brain-derived neurotrophic factor is reduced in Alzheimer's disease. Brain Res Mol Brain Res. 1997; 49:71-81. https://doi.org/10.1016/s0169-328x(97)00125-3.

25. Ginsberg SD, Alldred MJ, Counts SE, Cataldo AM, Neve RL, Jiang Y, et al. Microarray analysis of hippocampal CA1 neurons implicates early endosomal dysfunction during Alzheimer's disease progression. Biol Psychiatry. 2010; 68:885-93. https://doi.org/10.1016/j.biopsych.2010.05.030.

26. Michalski B, Fahnestock M. Pro-brain-derived neurotrophic factor is decreased in parietal cortex in Alzheimer's disease. Brain Res Mol Brain Res. 2003; 111:148-54. https://doi.org/10.1016/s0169328x(03)00003-2.

27. Peng S, Wuu J, Mufson EJ, Fahnestock M. Precursor form of brain-derived neurotrophic factor and mature brain-derived neurotrophic factor are decreased in the pre-clinical stages of Alzheimer's disease. J Neurochem. 2005; 93:1412-21. https://doi.org/10.1111/j.1471-4159.2005.03135.x.

28. Devi L, Ohno M. 7,8-dihydroxyflavone, a small-molecule TrkB agonist, reverses memory deficits and BACE1 elevation in a mouse model of Alzheimer's disease. Neuropsychopharmacology. 2012; 37:434-44. https://doi.org/10.1038/npp.2011.191.

29. Peng S, Garzon DJ, Marchese M, Klein W, Ginsberg SD, Francis BM, et al. Decreased brain-derived neurotrophic factor depends on amyloid aggregation state in transgenic mouse models of Alzheimer's disease. J Neurosci. 2009; 29:9321-9. https://doi.org/10.1523/jneurosci.4736-08.2009.

30. Bemelmans AP, Husson I, Jaquet M, Mallet J, Kosofsky BE, Gressens P. Lentiviral-mediated gene transfer of brain-derived neurotrophic factor is neuroprotective in a mouse model of neonatal 
excitotoxic challenge. J Neurosci Res. 2006; 83:50-60. https://doi.org/10.1002/jnr.20704.

31. Ma XC, Liu P, Zhang XL, Jiang WH, Jia M, Wang CX, et al. Intranasal Delivery of Recombinant AAV Containing BDNF Fused with HA2TAT: a Potential Promising Therapy Strategy for Major Depressive Disorder. Sci Rep. 2016; 6:22404. https://doi.org/10.1038/srep22404.

32. Blurton-Jones M, Kitazawa M, Martinez-Coria H, Castello NA, Müller FJ, Loring JF, et al. Neural stem cells improve cognition via BDNF in a transgenic model of Alzheimer disease. Proc Natl Acad Sci U S A. 2009; 106:13594-9. https://doi.org/10.1073/pnas.0901402106.

33. Eremenko E, Mittal K, Berner O, Kamenetsky N, Nemirovsky A, Elyahu Y, et al. BDNF-producing, amyloid $\beta$-specific CD4 T cells as targeted drug-delivery vehicles in Alzheimer's disease.

EBioMedicine. 2019; 43:424-34. https://doi.org/10.1016/j.ebiom.2019.04.019.

34. Makar TK, Trisler D, Eglitis MA, Mouradian MM, Dhib-Jalbut S. Brain-derived neurotrophic factor (BDNF) gene delivery into the CNS using bone marrow cells as vehicles in mice. Neurosci Lett. 2004; 356:215-9. https://doi.org/10.1016/j.neulet.2003.11.045.

35. Mitroshina EV, Yarkov RS, Mishchenko TA, Krut VG, Gavrish MS, Epifanova EA, et al. Brain-Derived Neurotrophic Factor (BDNF) Preserves the Functional Integrity of Neural Networks in the $\beta$ Amyloidopathy Model in vitro. Front Cell Dev Biol. 2020; 8:582. https://doi.org/10.3389/fcell.2020.00582.

36. Di Domenico F, Tramutola A, Perluigi M. Cathepsin D as a therapeutic target in Alzheimer's disease. Expert Opin Ther Targets. 2016; 20:1393-5. https://doi.org/10.1080/14728222.2016.1252334.

37. Suire CN, Leissring MA. Cathepsin D: A Candidate Link between Amyloid $\beta$-protein and Tauopathy in Alzheimer Disease. J Exp Neurol. 2021; 2:10-5.

38. Seira O, Del Río JA. Glycogen synthase kinase 3 beta (GSK3 $\beta$ ) at the tip of neuronal development and regeneration. Mol Neurobiol. 2014; 49:931-44. https://doi.org/10.1007/s12035-013-8571-y.

39. Beurel E, Grieco SF, Jope RS. Glycogen synthase kinase-3 (GSK3): regulation, actions, and diseases. Pharmacology \& therapeutics. 2015; 148:114-31. https://doi.org/10.1016/j.pharmthera.2014.11.016.

40. Reddy PH. Amyloid beta-induced glycogen synthase kinase $3 \beta$ phosphorylated VDAC1 in Alzheimer's disease: implications for synaptic dysfunction and neuronal damage. Biochim Biophys Acta. 2013; 1832:1913-21. https://doi.org/10.1016/j.bbadis.2013.06.012.

41. Salcedo-Tello P, Ortiz-Matamoros A, Arias C. GSK3 Function in the Brain during Development, Neuronal Plasticity, and Neurodegeneration. Int J Alzheimers Dis. 2011; 2011:189728. https://doi.org/10.4061/2011/189728.

42. Li Z, Tan F, Thiele CJ. Inactivation of glycogen synthase kinase-3beta contributes to brain-derived neutrophic factor/TrkB-induced resistance to chemotherapy in neuroblastoma cells. Mol Cancer Ther. 2007; 6:3113-21. https://doi.org/10.1158/1535-7163.Mct-07-0133.

43. Zhao S, Fu J, Liu X, Wang T, Zhang J, Zhao Y. Activation of Akt/GSK-3beta/beta-catenin signaling pathway is involved in survival of neurons after traumatic brain injury in rats. Neurol Res. 2012; 34:400-7. https://doi.org/10.1179/1743132812y.0000000025. 
44. Swerdlow RH, Burns JM, Khan SM. The Alzheimer's disease mitochondrial cascade hypothesis. J Alzheimers Dis. 2010; 20 Suppl 2:S265-79. https://doi.org/10.3233/jad-2010-100339.

45. Chen HH, Zhang N, Li WY, Fang MR, Zhang H, Fang YS, et al. Overexpression of brain-derived neurotrophic factor in the hippocampus protects against post-stroke depression. Neural Regen Res. 2015; 10:1427-32. https://doi.org/10.4103/1673-5374.165510.

Figures 


\section{(A) in vivo model}

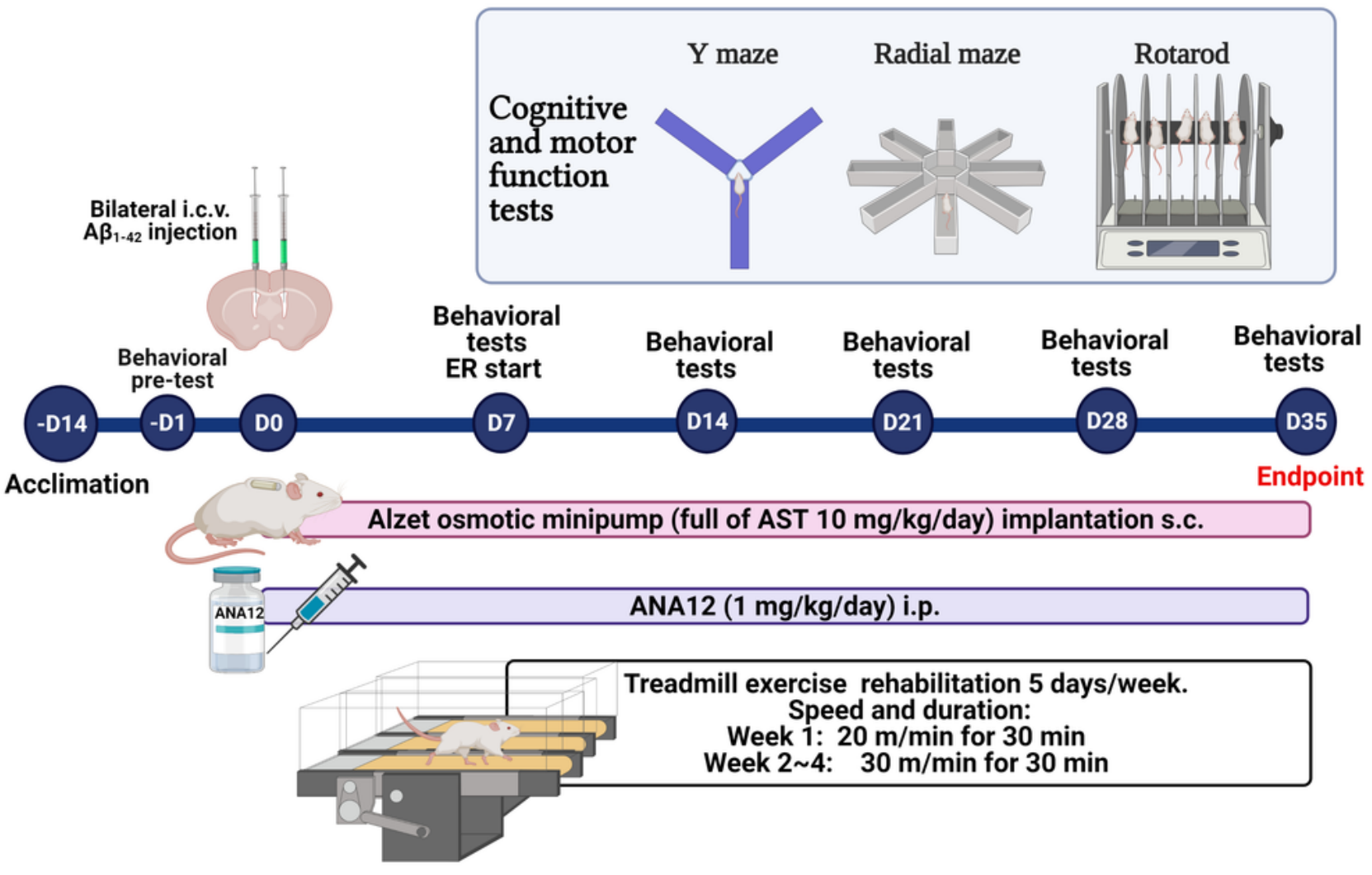

(B) in vitro model

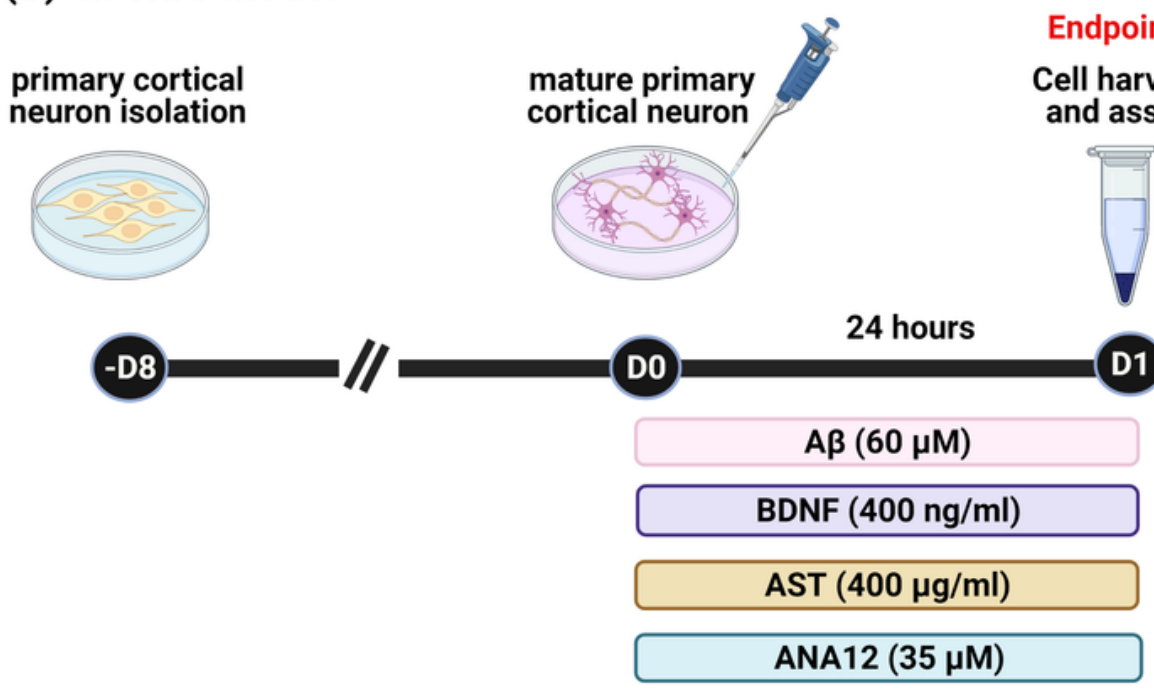

Endpoint

Cell harvest

1. MTT: Cell viability

2. Gel electrophoresis: DNA fragmentation

3. IF stain: synaptic plasticity 4. JC-1 stain: mitochondrial membrane potential 5. Sub-G1 stain: cell cycle (sub-G1 phase).

6. Western blot: pTrkB, pAKT, pGSK3 $\beta, \beta$-catenin, $\beta$-actin.

(created with BioRender.com)

\section{Figure 1}

Experimental design. (A) In the in vivo study, Wistar rats were allowed to acclimate to the environment for at least seven days. Then, rats were subjected to a battery of cognitive and motor function learning programs, including Y-maze, radial maze, and rotarod tests. One day before surgery, rats have performed the cognitive and motor function pre-test. On surgery day, the rat was anesthetized and placed onto a stereotaxic apparatus. Bilateral intracerebroventricular (i.c.v.) injections of A 1 1-42 (10 $\mu \mathrm{l} /$ ventricle) were 
performed using a Hamilton syringe. Meanwhile, the ALZET osmotic pump, containing full of AST, was implanted subcutaneously. The rat allowed to recovery from anesthesia, and the ANA-12 $(1 \mathrm{mg} / \mathrm{kg})$ was administered intraperitoneally immediately. Rats were returned to the standard housing. The ANA-12 $(1 \mathrm{mg} / \mathrm{kg} /$ day, i.p.) was administrated once per day to the endpoint of the experiments. Starting seven days later, some received four weeks of exercise rehabilitation (ER). (B) In the in vitro study, the primary cultured cortical neurons were prepared from embryonic day (D17-18) of Sprague-Dawley rat fetuses. Neuronal cultures were maintained for eight days in vitro before the various chemical treatments. After the medium was refreshed, the cortical neuron cultures were incubated with BDNF $(400 \mathrm{ng} / \mathrm{ml})$ and/or AST $(400 \mu \mathrm{g} / \mathrm{ml})$ and/or ANA12 $(35-60 \mu \mathrm{M})$ followed by exposure to $60 \mu \mathrm{M}$ aggregated Aß25-35 for 24 hours. The cell or cell lysate was harvested for cell viability (by MTT assay), mitochondrial membrane permeability and electrochemical potential (by JC-1 stain), DNA fragmentation (sub-G1 and DNA ladder assay), synaptic plasticity (by immunofluorescence stain), and pTrkB/pAkt/pGSK3 $\beta / \beta$-catenin signaling (by Western blot) measurement. 
(A)

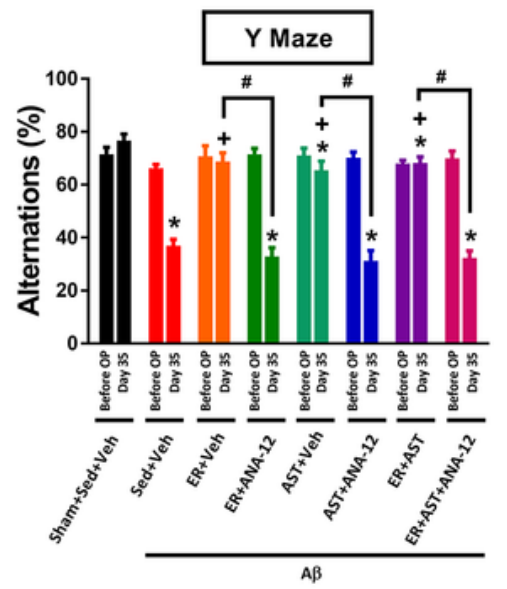

(C)

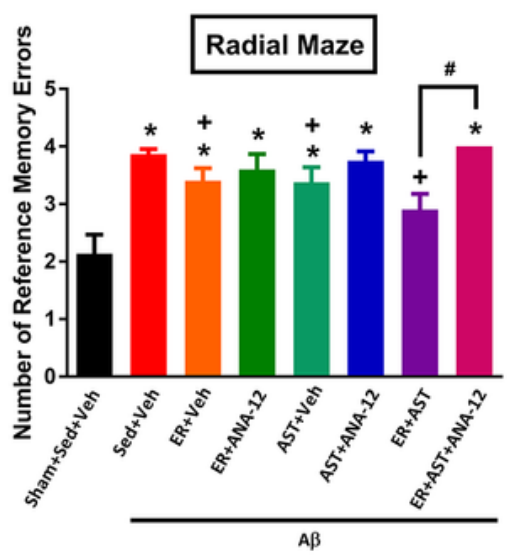

(E)

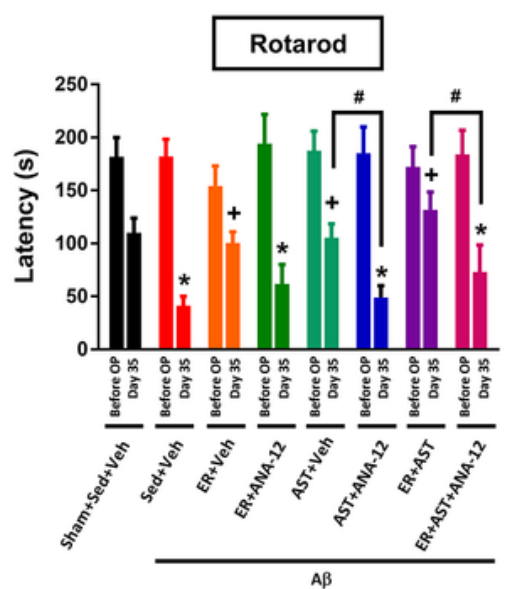

(B)

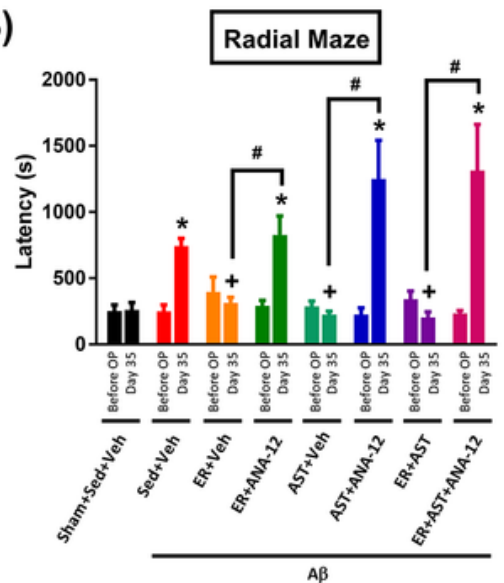

(D)

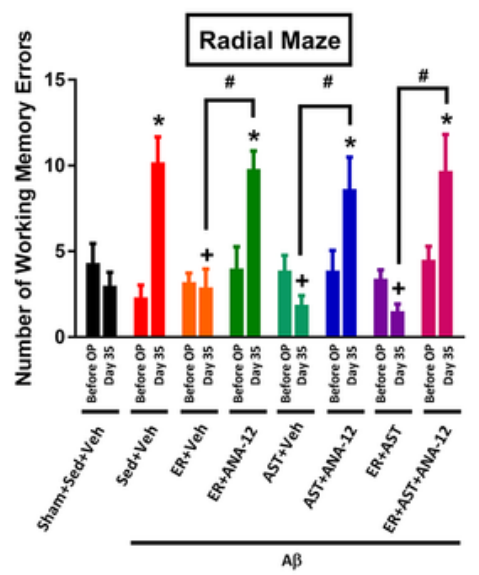

(F)

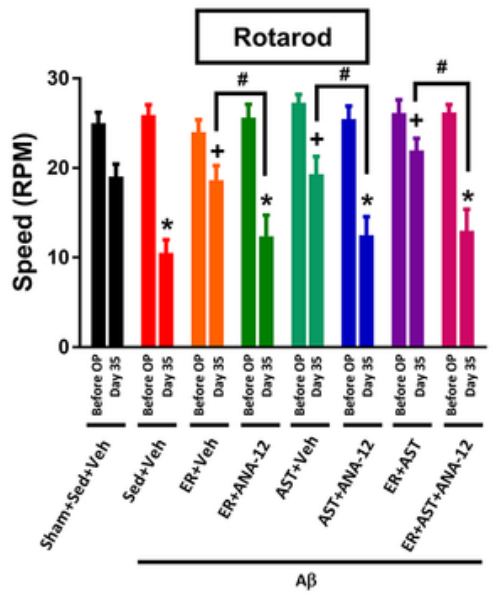

" $p<0.05$, compared with Sham + Sed $+V e h$

$+p<0.05$, compared with $A \beta+S e d+V e h$

\#p<0.05, compared with $A \beta+E R+V$ eh or $A \beta+A S T+V e h$ or $A \beta+E R+A S T$

Figure 2

Exercise rehabilitation (ER) and/or astragaloside (AST) reversed the motor and cognitive deficits by $A \beta$, which ANA12 wiped out any gains made from ER and/or AST. The spontaneous alternation in the Y-maze (A), the latency to elapse(B), numbers of reference memory errors(C), and numbers of working memory errors(D) in the radial maze tests, and the latency to fall off the rotarod (E) and the maximum speed reached during the test $(F)$ in the rotarod tests were performed. Data are presented as the means \pm SD 
$(n=10) .{ }^{*} p<0.05$, compared with Sham $+V e h+V e h$ group; $+p<0.05$, compared with $A \beta+V e h+V e h$ group; and $\# p<0.05, A \beta+A S T+V$ eh group vs. $A \beta+A S T+A N A 12$ group or $A \beta+E R+V$ eh group vs. $A \beta+E R+A N A 12$ group or $A \beta+B D N F+A S T$ group vs. $A \beta+B D N F+A S T+A N A 12$ group.

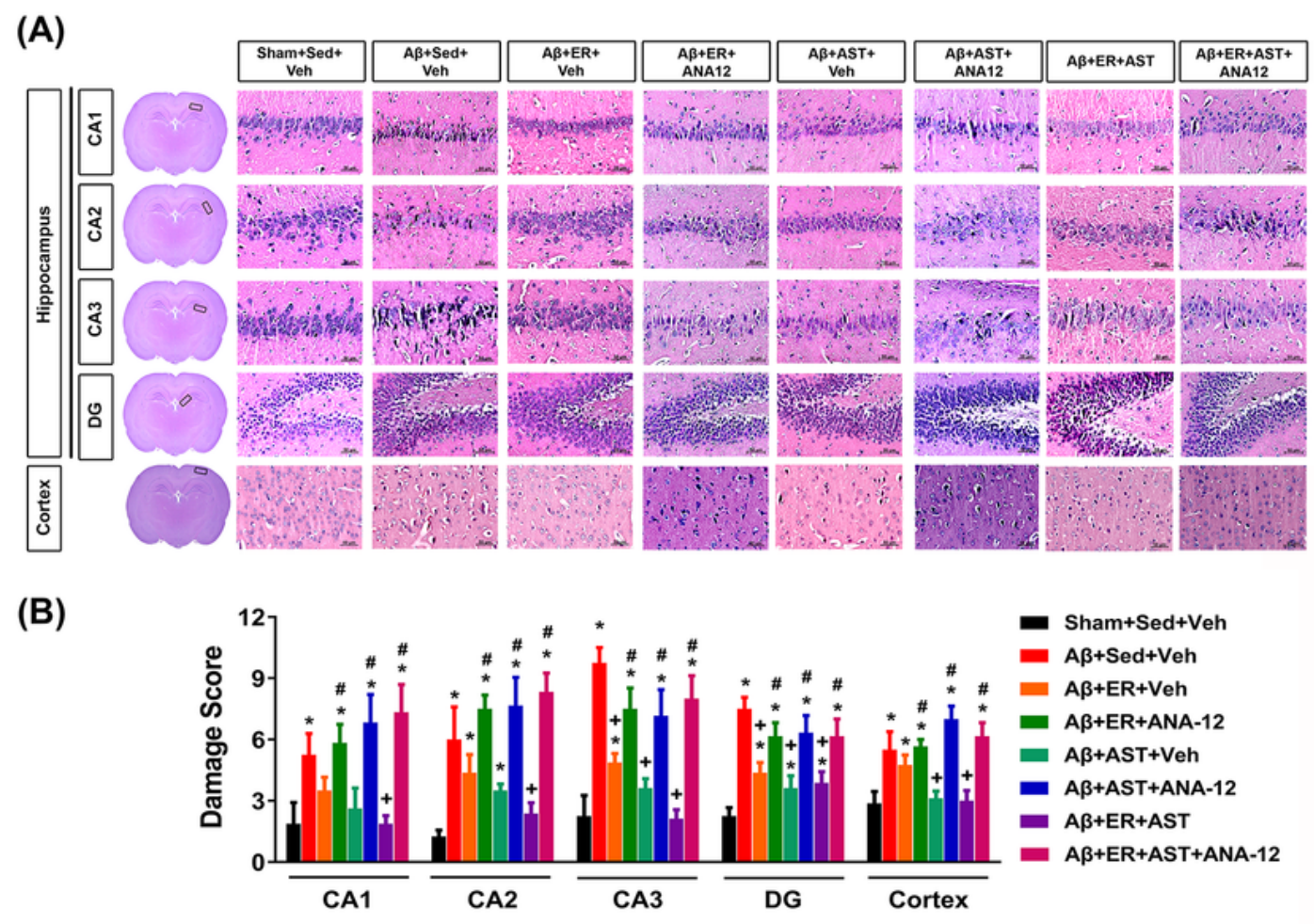

(C)

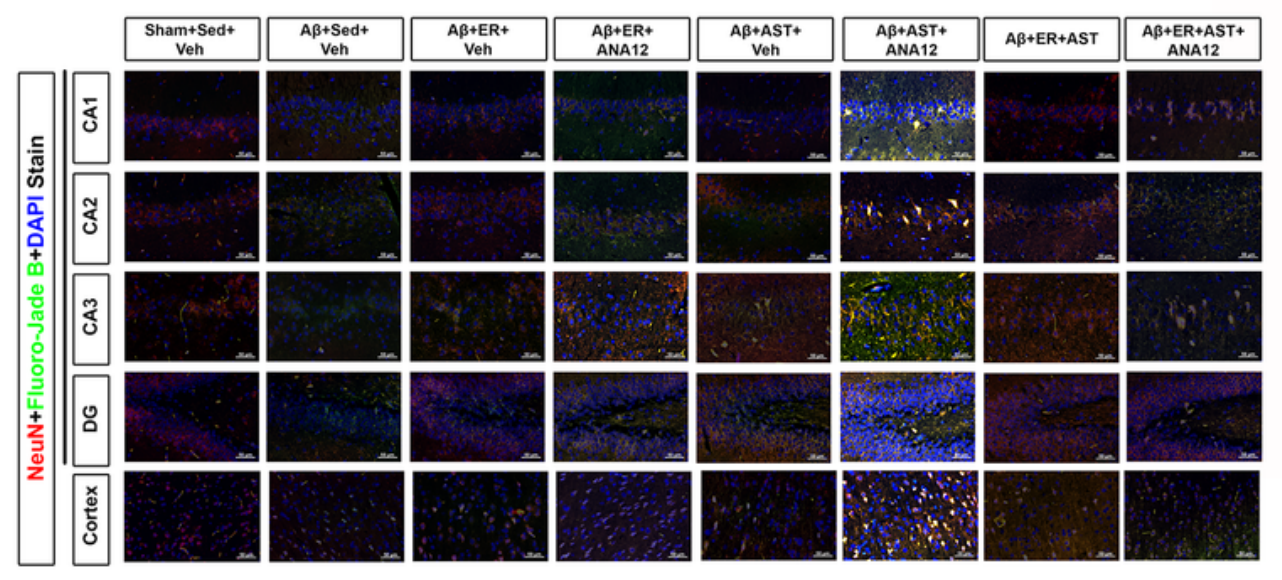

(D)

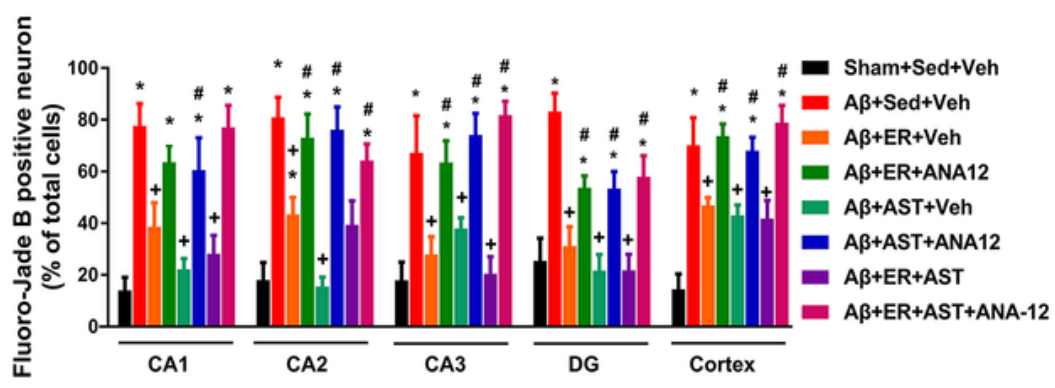

Figure 3

ER and/or AST reversed the hippocampal and cortical neuron damage and degeneration by $A \beta$, which can be eliminated by ANA12 in rats. Histology of hippocampus and cortex from different groups of rats 
using $\mathrm{H}$ \& E staining ( $\mathrm{A}$ and $\mathrm{B}$ ) and NeuN+Fluoro-Jade $\mathrm{B}+\mathrm{DAPI}$ staining ( $\mathrm{C}$ and $\mathrm{D})$. Representative photographs and quantification of different stainings were presented. Data are presented as the means \pm $S D(n=10) .{ }^{*} p<0.05$, compared with Sham $+V e h+V e h$ group; $+p<0.05$, compared with $A \beta+V e h+V e h$ group; $\# p<0.05, A \beta+A S T+V$ eh group vs. $A \beta+A S T+A N A 12$ group or $A \beta+E R+V$ eh group vs. $A \beta+E R+A N A 12$ group or $A \beta+B D N F+A S T$ group vs. $A \beta+B D N F+A S T+A N A 12$ group.

(A)

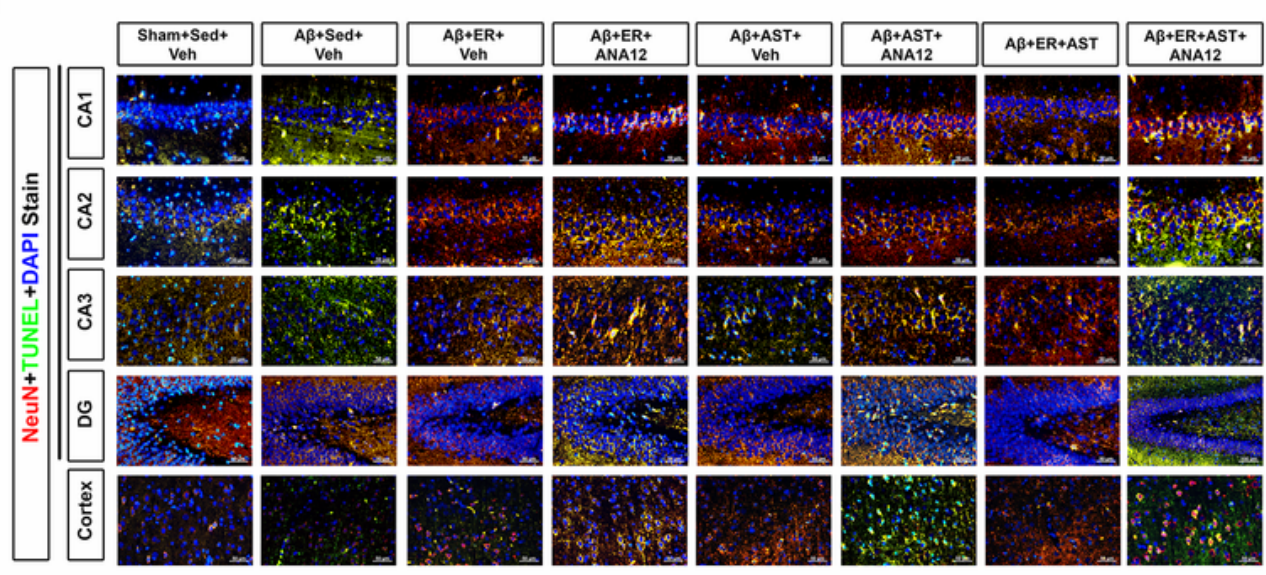

(B)

(C)
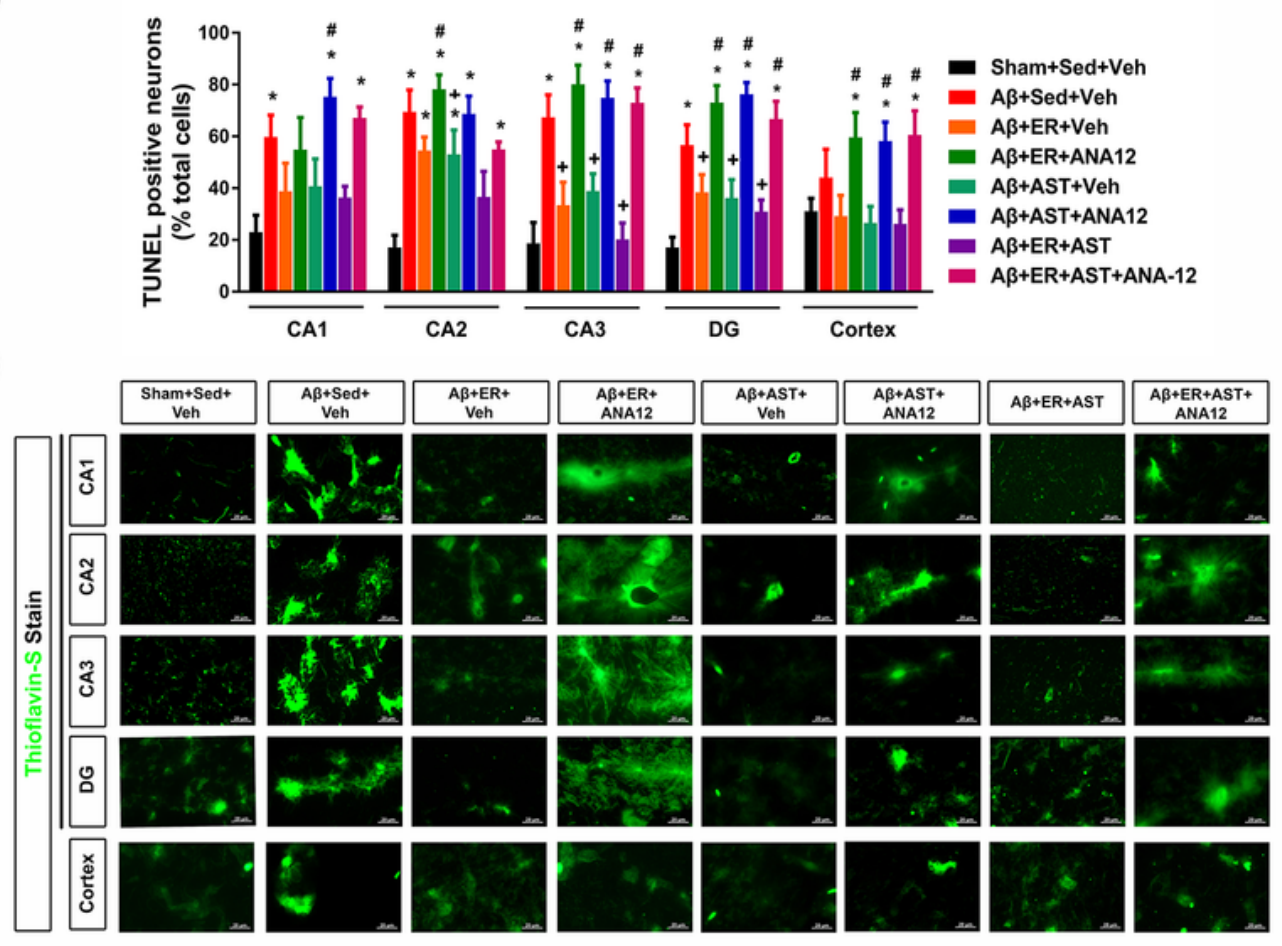

(D)

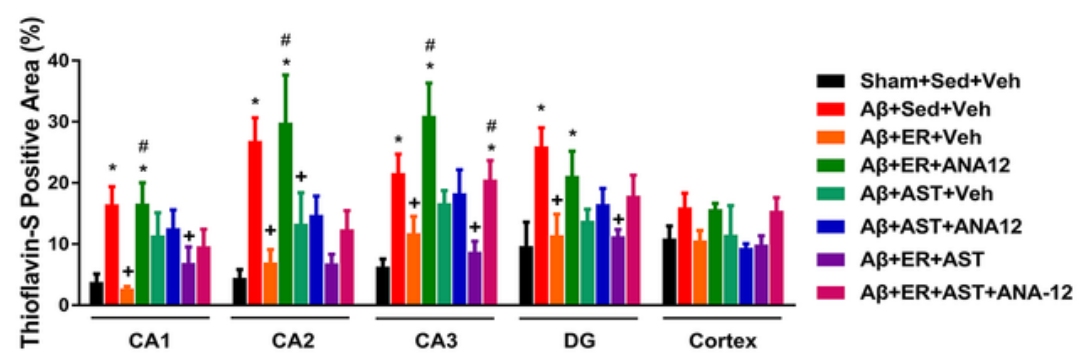

Figure 4 
ER and/or AST reversed the hippocampal and cortical neuron apoptosis and A $\beta$ accumulation by $A \beta$, which can be eliminated by ANA12 in rats. ER, AST, and ER combined with AST therapy inhibited Aßinduced neuronal apoptosis and $A \beta$ accumulation in an A 31 -42-treated rat by using NeuN/TUNEL immunofluorescence staining (A, B), and thioflavin-S stain (C, D)in different groups. Data are presented as the means $\pm S D(n=10)$. ${ }^{*}<0.05$, compared with Sham+Veh+Veh group; $+p<0.05$, compared with $A \beta+V e h+V e h$ group; $\# p<0.05$, $A \beta+A S T+V e h$ group vs. $A \beta+A S T+A N A 12$ group or $A \beta+E R+V$ eh group vs. $A \beta+E R+A N A 12$ group or $A \beta+B D N F+A S T$ group vs. $A \beta+B D N F+A S T+A N A 12$ group.

(A)

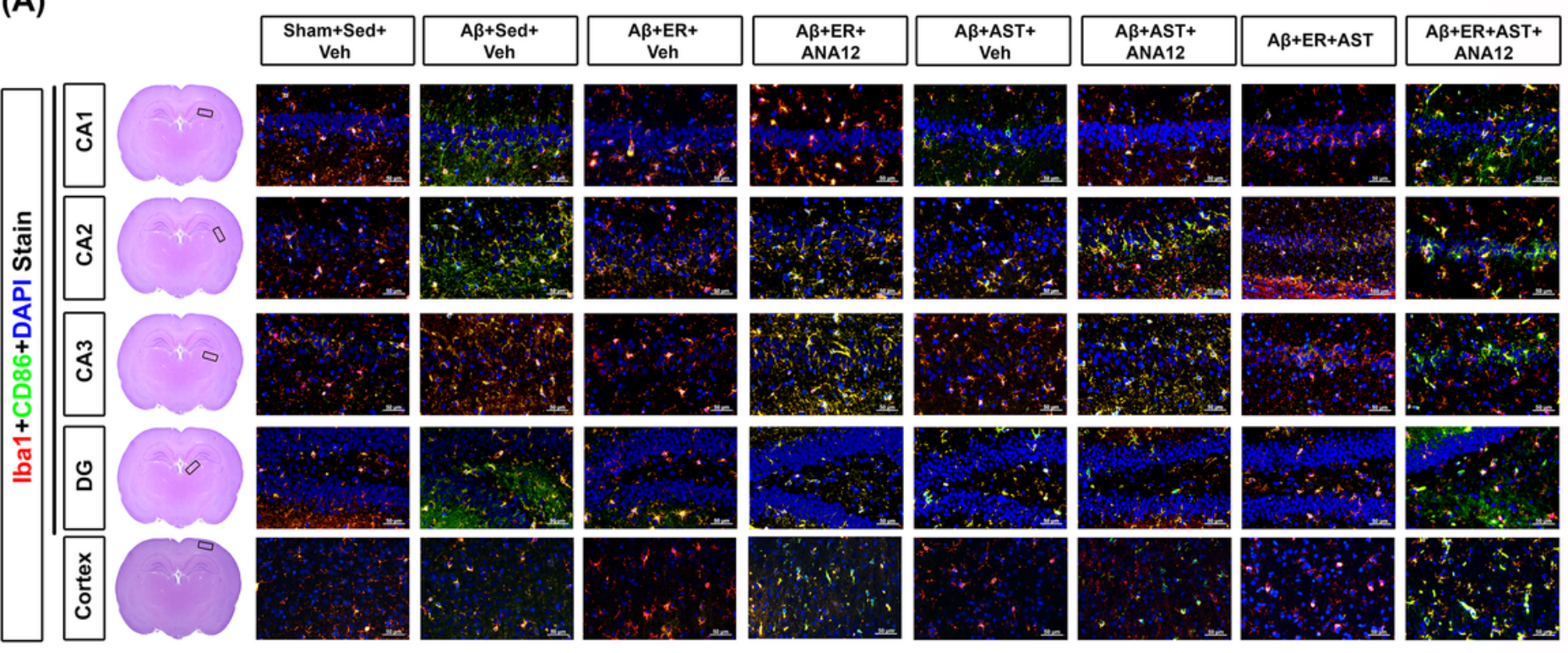

(B)

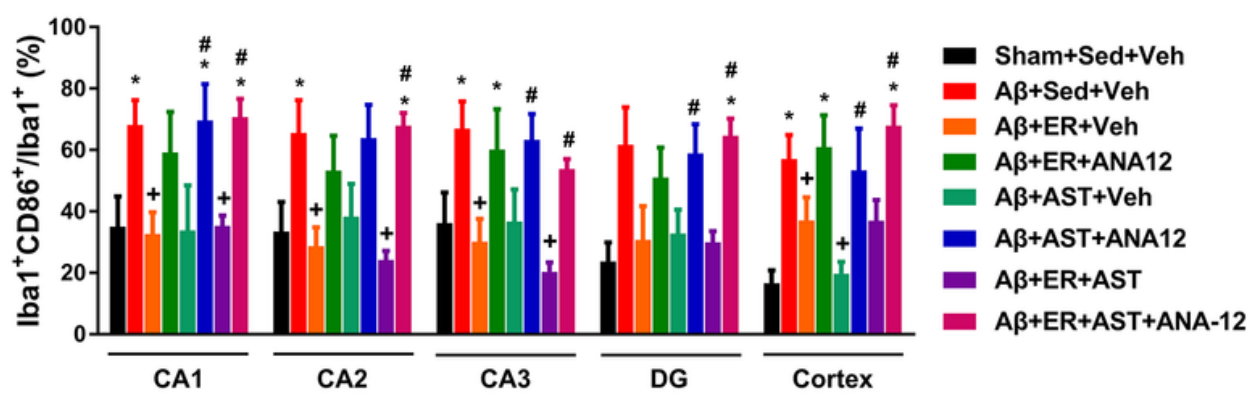

Figure 5

ER and/or AST reversed the hippocampal and cortical microgliosis by $A \beta$, which can be eliminated by ANA12 in rats ER, AST, and ER combined with AST therapy inhibited Aß-induced microglia polarization (as revealed by lba-1 and CD86 co-positive cells) in an AB1-42-treated rat by using immunofluorescence staining in different groups. Data are presented as the means $\pm S D(n=10) .{ }^{*} p<0.05$, compared with Sham+Veh+Veh group; $+p<0.05$, compared with $A \beta+V e h+V$ eh group; $\# p<0.05, A \beta+A S T+V e h$ group vs. $A \beta+A S T+A N A 12$ group or $A \beta+E R+V e h$ group vs. $A \beta+E R+A N A 12$ group or $A \beta+B D N F+A S T$ group vs. $A \beta+B D N F+A S T+A N A 12$ group. 
(A)
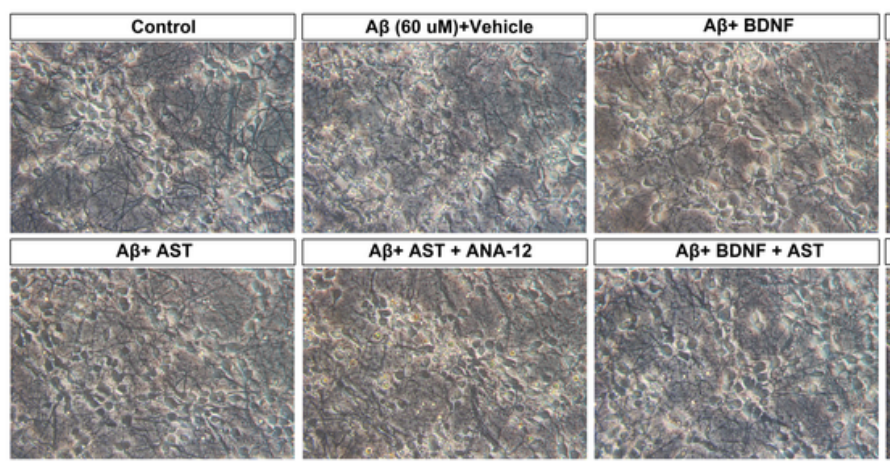

(C)
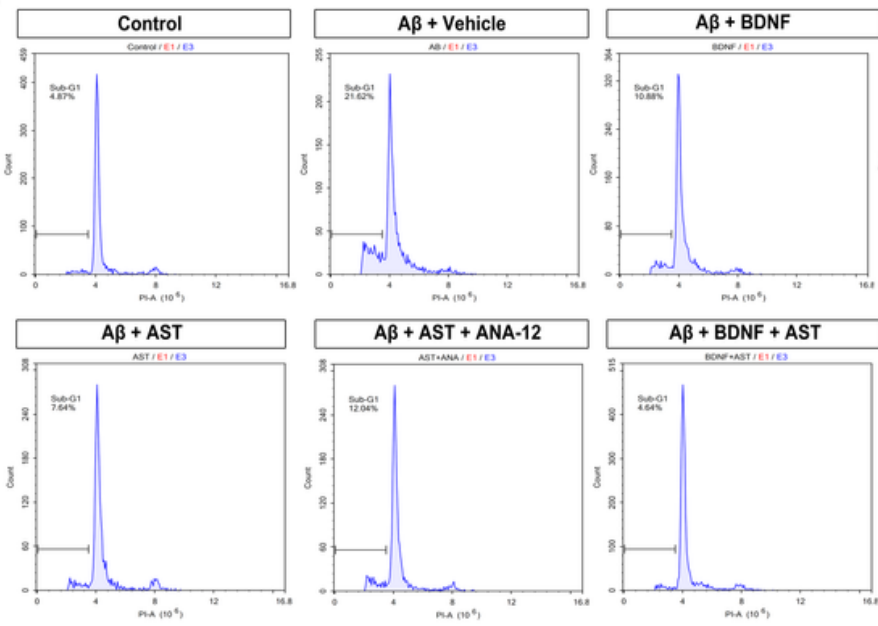

(F)
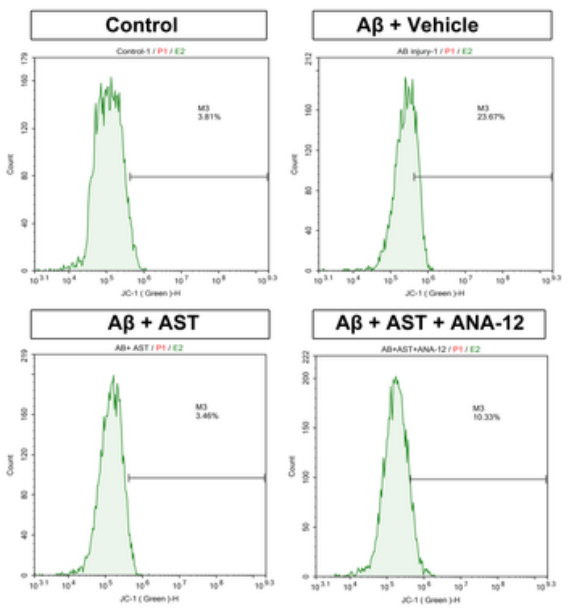
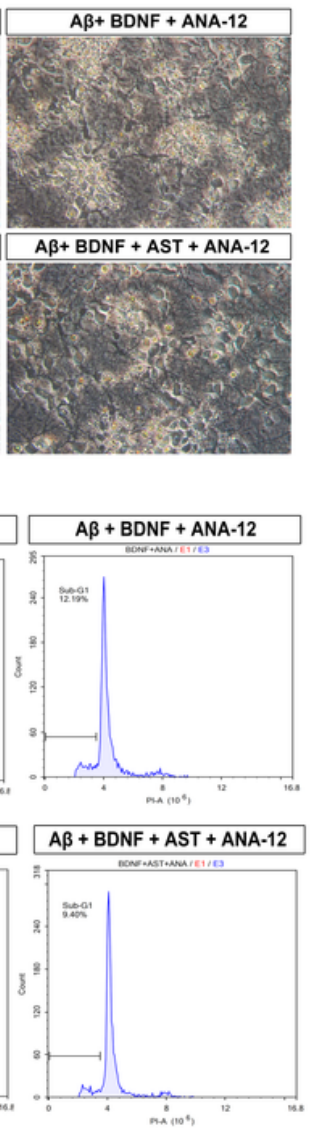

(B)

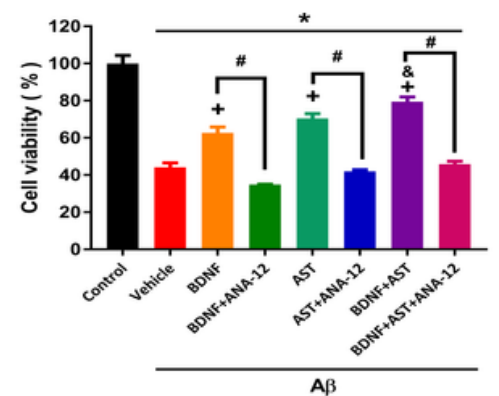

(D)

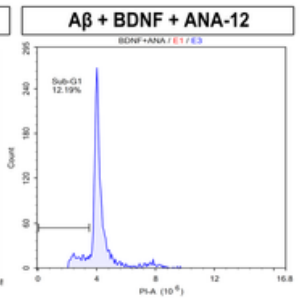

(E)
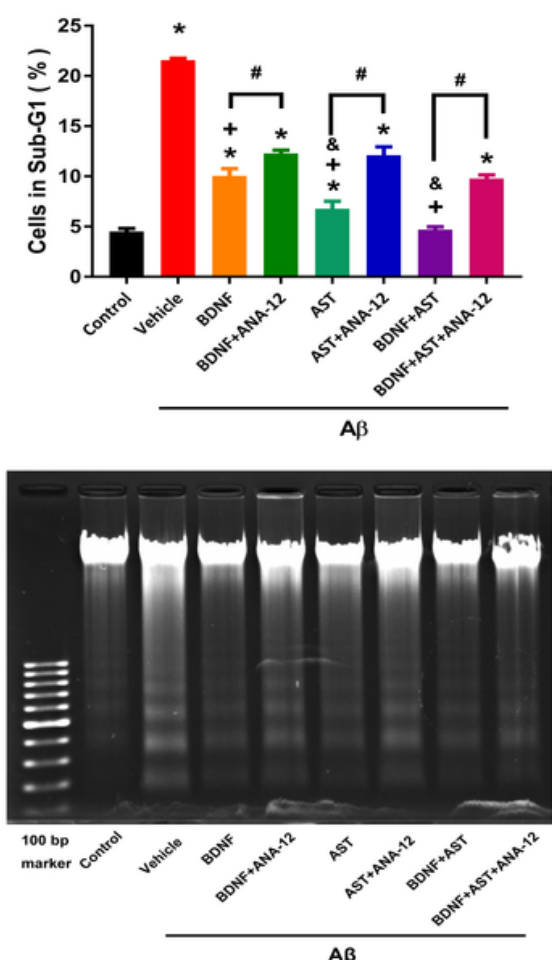

(G)

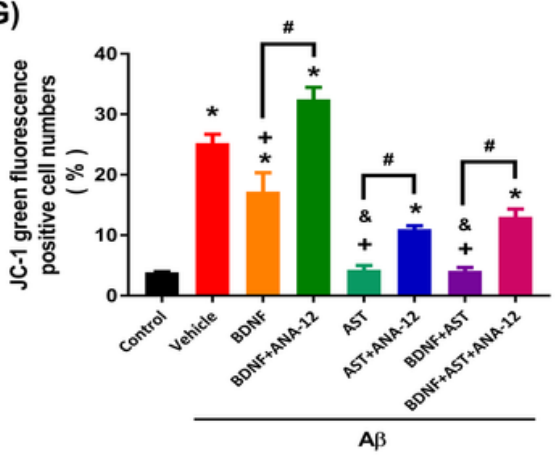

\section{Figure 6}

Local application of BDNF and/or AST reversed the dysmorphological changes, decreased cell viability, apoptosis, and mitochondrial distress by $A \beta$ in cortical neurons which could be eliminated by ANA12. BDNF and/or AST inhibited A $\beta$-induced (A) morphological changes, (B) decreased cell viability, (C, D) increased apoptosis (sub-G1 phase), (E) DNA fragmentation, $(F, G)$ increased percentage of JC-1 green fluorescence positive cells number (indicates mitochondrial distress) in cultured primary cortical neurons. Data are presented as the means \pm standard deviation of five independent experiments. *p<0.05, 
compared with Control+Veh group; $+p<0.05$, compared with $A \beta+$ Veh group; $\# p<0.05, A \beta+B D N F$ vs. $A \beta+B D N F+A N A 12$ or $A \beta+A S T$ vs. $A \beta+A S T+A N A 12$ or $A \beta+B D N F+A S T$ vs. $A \beta+B D N F+A S T+A N A 12$. $\& p<0.05, A \beta+B D N F$ vs. $A \beta+B D N F+A S T$ or $A \beta+A S T$ vs. $A \beta+B D N F+A S T$.

(A) MAP2+Synaptophysin+DAPI

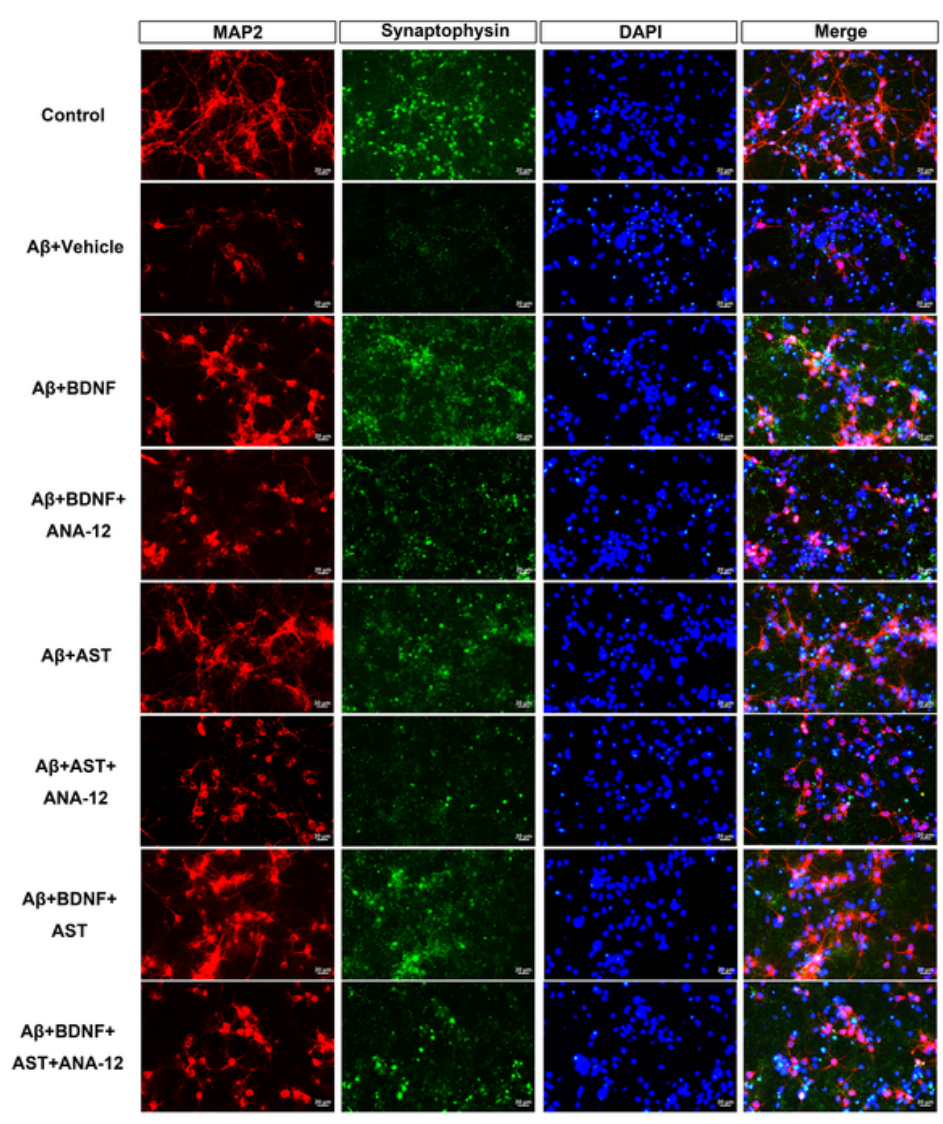

(B)

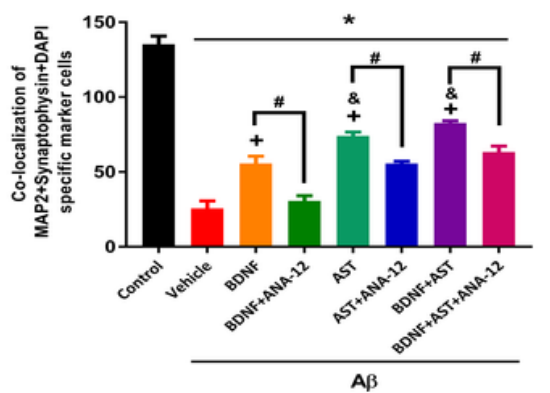

(F) Brain tissues
(C) Primary cortical neurons

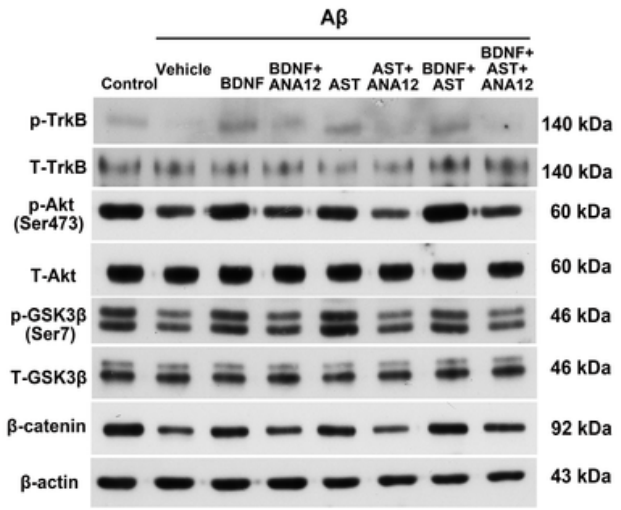

(D) Primary cortical neurons

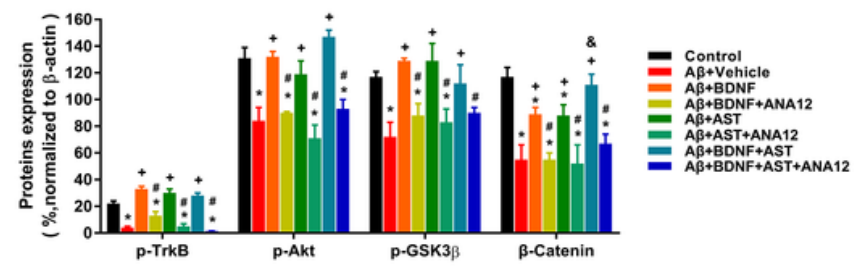

(E) Brain tissues
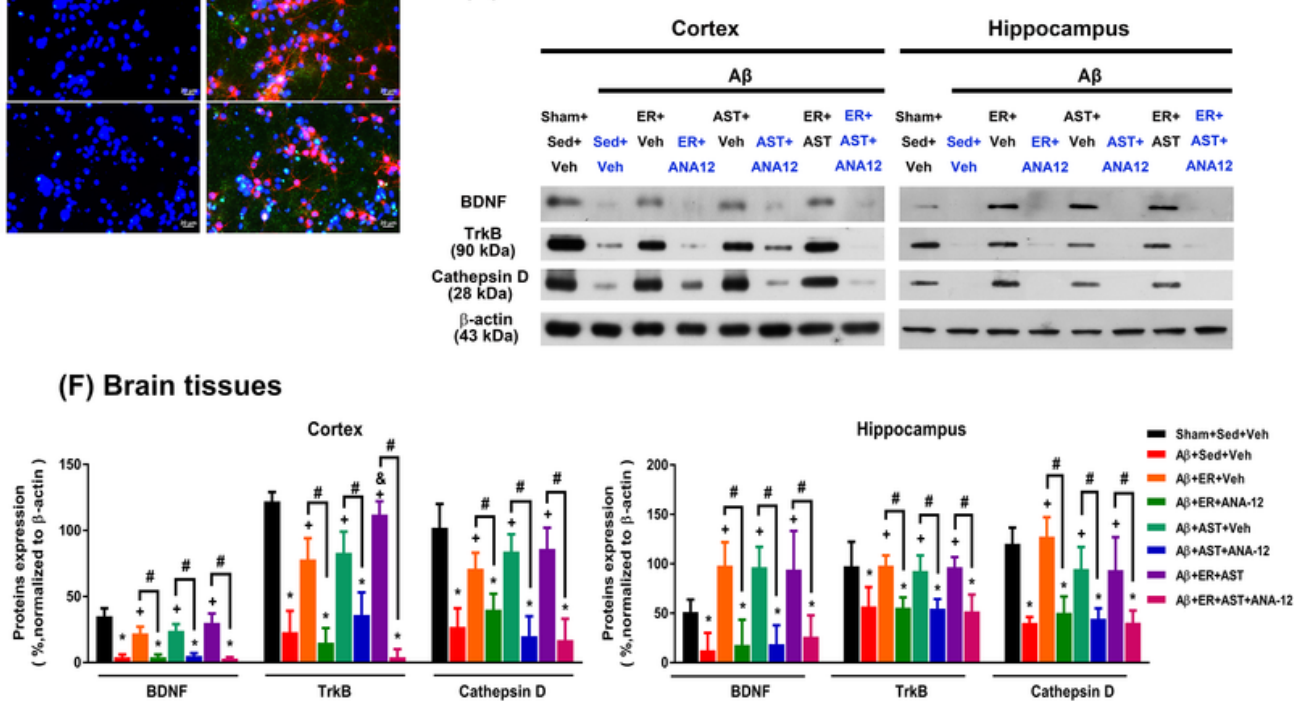

Figure 7

Topical application of BDNF and/or AST reversed the A $\beta$-induced synaptotoxicity, $p$-TrkB, $p$-GSK3 $\beta$, and $\beta$ catenin downregulation in cortical neurons and BDNF, TrkB, and cathepsin D expression in brain tissue which could be eliminated by ANA12. BDND and/or AST attenuated A $\beta$-induced $(A, B)$ synaptotoxicity (increased loss of both MAP-2 and synaptophysin), (C, D) phosphor-TrkB (p-TrkB), total-TrkB (t-TrkB), phosphor-Akt (p-Akt), total-Akt (t-Akt), phosphor-GSK3 $\beta(p-G S K \beta)$, and $\beta$-catenin expression downregulation in primary cortical neuronal cultures. The data are from five independent experiments. 
${ }^{*} p<0.05$, compared with Control+Veh group; $+p<0.05$, compared with $A \beta+V e h$ group; $\# p<0.05, A \beta+B D N F$ vs. $A \beta+B D N F+A N A 12$ or $A \beta+A S T$ vs. $A \beta+A S T+A N A 12$ or $A \beta+B D N F+A S T$ vs. $A \beta+B D N F+A S T+A N A 12$.

$\& p<0.05, A \beta+B D N F$ vs. $A \beta+B D N F+A S T$ or $A \beta+A S T$ vs. $A \beta+B D N F+A S T$. (E) Represent Western blotting of $B D N F, T r k B$, and cathepsin $D$ expression in cortical and hippocampal tissues. ( $F$ ) Densitometry analyses of BDNF, TrkB, and cathepsin D expression normalized to $\beta$-actin. Data present the means $\pm S D(n=6)$. ${ }^{*} p<0.05$, compared with Sham+Sed+Veh group; $+p<0.05$, compared with $A \beta+S e d+V e h$ group; $\# p<0.05$, $A \beta+E R+V$ eh group vs. $A \beta+E R+A N A 12$ group or $A \beta+A S T+V$ eh group vs. $A \beta+A S T+A N A 12$ group or $A \beta+B D N F+A S T$ group vs. $A \beta+B D N F+A S T+A N A 12$ group. \& $p<0.05, A \beta+E R+V e h$ vs. $A \beta+E R+A S T$.

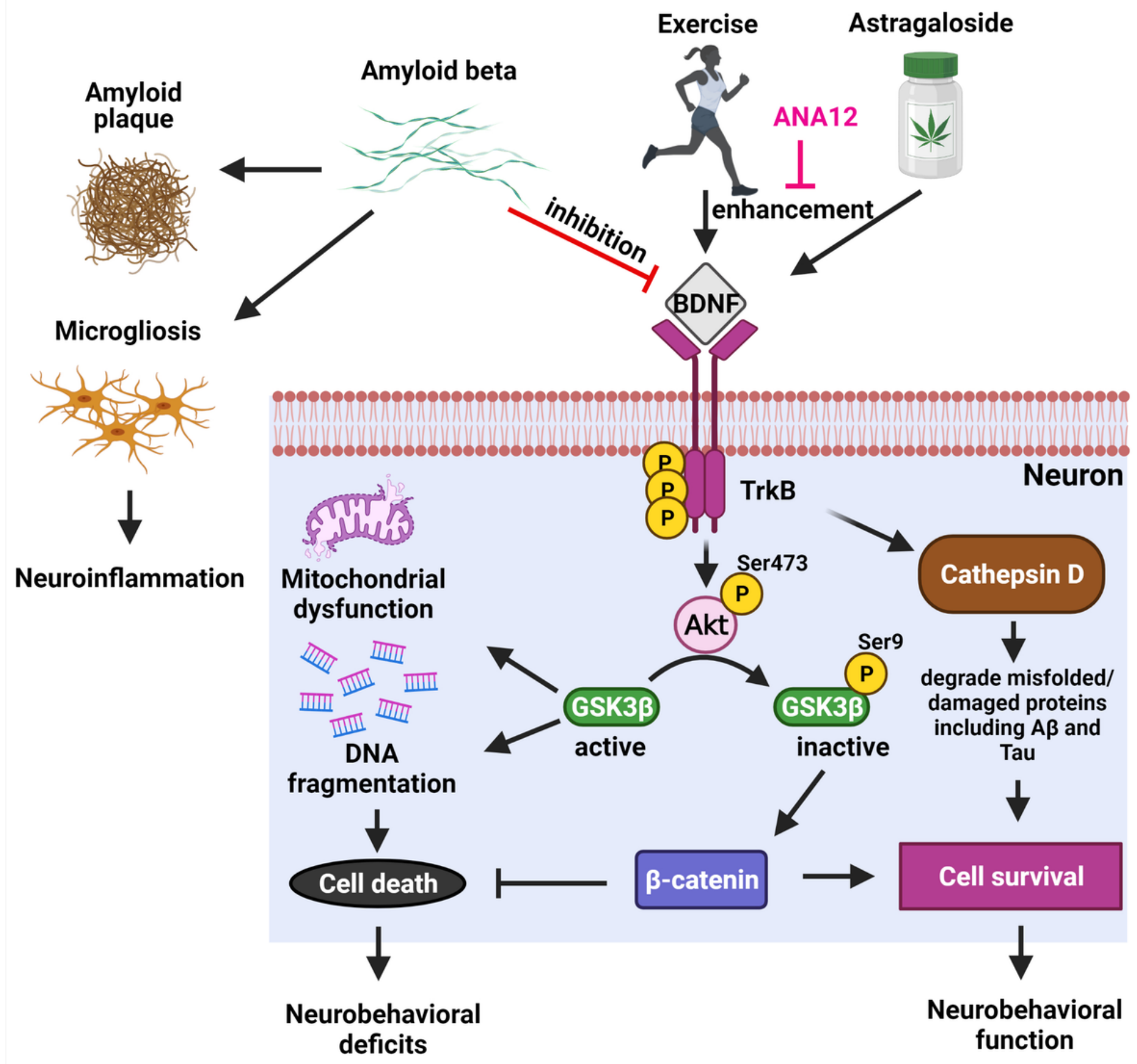

Figure 8 
Proposed model of BDNF/TrkB signaling deficits and mitochondrial dysfunction in the neuron. BDNF interacts with TrkB to activate p-Akt phosphorylation (at residue serine 473), which phosphorylates the serine 9 residue of GSK3 $\beta$ (GSK3 $\beta$ Ser9), and leads to GSK3 $\beta$ inactivation and stabilization of $\beta$-catenin expression, and then improves cell survival. Intracerebroventricularly microinjected $A \beta$ in rats might cause neurobehavioral disorders as well as neurodegeneration via suppressing cortical and hippocampal levels of $B D N F / T r k B /$ cathepsin $D$ signaling pathway. In vitro studies revealed that local application of $A \beta$ might induce cortical cell death via inhibiting BDNF/TrkB/p-Akt/p-GSK3 $/ \beta$-catenin signaling pathway. In rats, exercise rehabilitation and/or AST therapy significantly ameliorated the A $\beta$-induced neurological injury via normalizing the cortical and hippocampal levels of BDNF/TrkB/cathepsin D, which can be abolished by ANA12. Additionally, local application of BDNF (to mimick exercise effects) and/or AST also ameliorated $A \beta$-induced neuronal injury by normalizing the cellular levels of BDNF/TrkB/p-Akt/ pGSK3 $\beta$ / $\beta$-catenin, which can also be antagonized by ANA12. 